\title{
NILPOTENT ADMISSIBLE INDIGENOUS BUNDLES VIA CARTIER OPERATORS IN CHARACTERISTIC THREE
}

\author{
YUICHIRO HoshI
}

\begin{abstract}
In the present paper, we study the p-adic Teichmüller theory in the case where $p=3$. In particular, we discuss nilpotent admissible/ordinary indigenous bundles over a projective smooth curve in characteristic three. The main result of the present paper is a characterization of the supersingular divisors of nilpotent admissible/ordinary indigenous bundles in characteristic three by means of various Cartier operators. By means of this characterization, we prove that, for every nilpotent ordinary indigenous bundle over a projective smooth curve in characteristic three, there exists a connected finite étale covering of the curve on which the indigenous bundle is not ordinary. We also prove that every projective smooth curve of genus two in characteristic three is hyperbolically ordinary. These two applications yield negative, partial positive answers to basic questions in the p-adic Teichmüller theory, respectively.
\end{abstract}

\section{CONTENTS}

Introduction.

$\S 1$. Construction of a dormant indigenous bundle ...............

\$2. The dormant trivialization of the Schwarz torsor..............

$\$ 3 . \quad$ Local criteria ........................................... 700

$\S 4$. Indigenous bundles arising from squares................. 705

§5. Nilpotent admissible indigenous bundles via Cartier operators .. 710

$\S 6$. The case of genus two ............................... 714

$\S$ A. Cartier operator associated to a square-trivialized invertible sheaf........................................... 720

$\S$ B. The Hasse bundle of a nilpotent admissible indigenous bundle 725

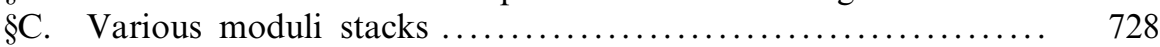

References........................................... 730

2010 Mathematics Subject Classification. $14 \mathrm{G} 17$.

Key words and phrases. p-adic Teichmüller theory, nilpotent admissible indigenous bundle, nilpotent ordinary indigenous bundle, Cartier operator.

Received October 15, 2014; revised May 26, 2015. 


\section{Introduction}

In the present paper, we study the p-adic Teichmüller theory established by S. Mochizuki [cf. [6], [7]] in the case where $p=3$. In particular, we discuss nilpotent admissible/ordinary indigenous bundles over a projective smooth curve in characteristic three. In the Introduction, let $p$ be an odd prime number, $g \geq 2$ an integer, $S$ a connected noetherian scheme of characteristic $p$ [i.e., over $\mathbf{F}_{p}$ ], and $f: X \rightarrow S$ a projective smooth curve [i.e., a morphism which is projective, smooth, geometrically connected, and of relative dimension one] of genus $g$ over $S$. Write $f^{F}: X^{F} \rightarrow S$ for the projective smooth curve over $S$ obtained by basechanging $X \rightarrow S$ via the absolute Frobenius morphism of $S$ and $\Phi: X \rightarrow X^{F}$ for the relative Frobenius morphism over $S$. We use the notation " $\omega$ " (respectively, " $\tau$ ") to denote the relative cotangent (respectively, tangent) sheaf.

First, let us recall the notion of an indigenous bundle and some properties on an indigenous bundle. We shall say that a pair

$$
\left(\pi: P \rightarrow X, \nabla_{P}\right)
$$

consisting of a $\mathbf{P}^{1}$-bundle $\pi: P \rightarrow X$ over $X$ and a connection $\nabla_{P}$ on $P$ relative to $X / S$ is an indigenous bundle over $X / S$ if there exists a [uniquely determined-cf. [6], Chapter I, Proposition 2.4, p. 1004] section [i.e., the Hodge section] $\sigma: X \rightarrow P$ of $\pi: P \rightarrow X$ such that the Kodaira-Spencer homomorphism $\sigma^{*} \omega_{P / X} \rightarrow \omega_{X / S}$ at $\sigma$ relative to $\nabla_{P}$ [i.e., the homomorphism obtained by differentiating $\sigma$ by means of $\nabla_{P}$ ] is an isomorphism [cf. [6], Chapter I, Definition 2.2, pp. 1002-1003]. The notion of an indigenous bundle was introduced and studied by R. C. Gunning [cf. [2], §2] and enables one to understand the theory of uniformization of [algebraic] Riemann surfaces in a somewhat more algebraic setting.

Let $\left(\pi: P \rightarrow X, \nabla_{P}\right)$ be an indigenous bundle over $X / S$. Then the connection $\nabla_{P}$ on $P$ determines a horizontal homomorphism [i.e., the p-curvature]

$$
\mathscr{P}: \Phi^{*} \tau_{X^{F} / S} \rightarrow \mathscr{A} d(P) \stackrel{\text { def }}{=} \pi_{*} \tau_{P / X} .
$$

We shall say that the indigenous bundle $\left(\pi: P \rightarrow X, \nabla_{P}\right)$ is nilpotent (respectively, admissible; dormant) if the square of $\mathscr{P}$ is zero (respectively, the zero locus of $\mathscr{P}$ is empty; $\mathscr{P}=0)$ [cf. [6], Chapter II, Definition 2.4, p. 1030 (respectively, [6], Chapter II, Definition 2.4, p. 1030; [7], Chapter II, Definition 1.1, p. 127)]. Moreover, we shall refer to the composite of the $p$-curvature $\mathscr{P}$ and the surjection $\mathscr{A} d(P) \rightarrow \tau_{X / S}$ determined by the Hodge section of $\left(\pi: P \rightarrow X, \nabla_{P}\right)$ as the square Hasse invariant of $\left(\pi: P \rightarrow X, \nabla_{P}\right)$ [cf. [6], Chapter II, Proposition 2.6, (1), p. 1032]. Then, by means of this square Hasse invariant, one may define the Frobenius on $\mathbf{R}^{1} f_{*} \tau_{X / S}$ induced by $\left(\pi: P \rightarrow X, \nabla_{P}\right)$ [cf. the discussion following [6], Chapter II, Lemma 2.11, pp. 1036-1037]. We shall say that the indigenous bundle $\left(\pi: P \rightarrow X, \nabla_{P}\right)$ is ordinary if the Frobenius on $\mathbf{R}^{1} f_{*} \tau_{X / S}$ induced by $\left(\pi: P \rightarrow X, \nabla_{P}\right)$ is an isomorphism [cf. [6], Chapter II, Definition 3.1, p. 1044]. A nilpotent admissible/ordinary indigenous bundle plays a central role in the "classical" p-adic Teichmüller theory, i.e., the p-adic Teichmüller theory discussed in [not [7] but] [6]. 
First, we verify the following uniqueness of a dormant indigenous bundle in characteristic three [cf. Theorem 2.1, Corollary 2.4]:

THEOREM A. In the notation introduced at the beginning of the Introduction, suppose that $p=3$. Then there exists a unique dormant indigenous bundle over $X / S$. In particular, there exists a natural bijection between

- $H^{0}\left(S, f_{*} \omega_{X / S}^{\otimes 2}\right)=H^{0}\left(X, \omega_{X / S}^{\otimes 2}\right)$ and

- the set of isomorphism classes of indigenous bundles over $X / S$ such that, for $\theta \in H^{0}\left(S, f_{*} \omega_{X / S}^{\otimes 2}\right)$, the dormant locus in $S$ of the indigenous bundle over $X / S$ corresponding to $\theta$ coincides with the zero locus in $S$ of $\theta$.

If an indigenous bundle $\left(\pi: P \rightarrow X, \nabla_{P}\right)$ over $X / S$ is nilpotent admissible, then there exist an invertible sheaf $\mathscr{H}$ on $X$ and a global section $\chi$ of $\mathscr{H}$ such that $\mathscr{H}^{\otimes 2} \cong \mathscr{H}^{\circ} m_{\mathcal{O}_{X}}\left(\Phi^{*} \tau_{X^{F} / S}, \tau_{X / S}\right)$, and, moreover, the square of $\chi$ coincides with the square Hasse invariant of $\left(\pi: P \rightarrow X, \nabla_{P}\right)$ [cf. [6], Chapter II, Proposition 2.6, (3), p. 1032]. We shall refer to $\chi$ as the Hasse invariant of $\left(\pi: P \rightarrow X, \nabla_{P}\right)$ [cf. [6], Chapter II, Proposition 2.6, (3), p. 1032] and to the zero locus of the Hasse invariant as the supersingular divisor of $\left(\pi: P \rightarrow X, \nabla_{P}\right)$ [cf. [6], Chapter II, Proposition 2.6, (3), p. 1032]. The supersingular divisor is an important invariant of a nilpotent admissible indigenous bundle; for instance, if $S$ is reduced, then the isomorphism class of a nilpotent admissible indigenous bundle over $X / S$ is completely determined by the supersingular divisor [cf. [6], Chapter II, Proposition 2.6, (4), p. 1032]. The main result of the present paper is a characterization of the supersingular divisors of nilpotent admissible/ordinary indigenous bundles in characteristic three by means of various Cartier operators.

In order to present the main result of the present paper, let us recall some notions related to the Cartier operator. Let $(\mathscr{L}, \Theta)$ be a square-trivialized invertible sheaf on $X$, i.e., a pair consisting of an invertible sheaf $\mathscr{L}$ on $X$ and a trivialization $\Theta$ of the square of $\mathscr{L}$ [cf. Definition A.3]. Then the [usual] Cartier operator $\Phi_{*} \omega_{X / S} \rightarrow \omega_{X^{F} / S}$, together with the trivialization $\Theta$, determines a homomorphism of $\mathcal{O}_{S}$-modules

$$
C_{(\mathscr{L}, \Theta)}: f_{*}\left(\mathscr{L} \otimes_{\mathcal{O}_{X}} \omega_{X / S}\right) \rightarrow f_{*}^{F}\left(\mathscr{L}^{F} \otimes_{\mathcal{O}_{X} F} \omega_{X^{F} / S}\right)
$$

- where we write $\mathscr{L}^{F}$ for the invertible sheaf on $X^{F}$ obtained by pulling back $\mathscr{L}$ via the morphism $X^{F} \rightarrow X$ induced by the absolute Frobenius morphism of $S$. We shall refer to this homomorphism as the Cartier operator associated to $(\mathscr{L}, \Theta)$ [cf. Definition A.4]. On the other hand, the morphism $X^{F} \rightarrow X$ induced by the absolute Frobenius morphism of $S$ determines a Frobenius-semi-linear homomorphism

$$
f_{*}\left(\mathscr{L} \otimes_{\mathcal{O}_{X}} \omega_{X / S}\right) \rightarrow f_{*}^{F}\left(\mathscr{L}^{F} \otimes_{\mathcal{O}_{X} F} \omega_{X^{F} / S}\right) .
$$

For a global section $u$ of $\mathscr{L} \otimes_{\mathcal{O}_{X}} \omega_{X / S}$, we shall write $u^{F}$ for the global section of $\mathscr{L}^{F} \otimes_{\mathcal{O}_{X} F} \omega_{X^{F} / S}$ obtained by forming the image of $u$ via this Frobenius-semi- 
linear homomorphism. We shall say that a global section $u$ of $\mathscr{L} \otimes_{\mathcal{O}_{X}} \omega_{X / S}$ is a normalized Cartier eigenform associated to $(\mathscr{L}, \Theta)$ if $u$ defines a relative effective Cartier divisor of $X / S$, and, moreover, $C_{(\mathscr{L}, \Theta)}(u)=-u^{F}$ [cf. Definition A.8, (i)]. (ii)]:

A part of the main result of the present paper is as follows [cf. Theorem 5.2,

THEOREM B. In the notation introduced at the beginning of the Introduction, suppose that $p=3$. Let $D$ be a relative effective Cartier divisor of $X / S$. Then it holds that $D$ is the supersingular divisor of a nilpotent admissible (respectively, nilpotent ordinary) indigenous bundle over $X / S$ if and only if $D$ is of CE-type (respectively, of CEO-type) [cf. Definition 5.1, (iii)], i.e., there exist an invertible sheaf $\mathscr{L}$ on $X$, a trivialization $\Theta$ of the square of $\mathscr{L}$, and a global section $\chi$ of $\mathscr{L} \otimes_{\mathcal{O}_{X}} \omega_{X / S}$ such that the following two (respectively, three) conditions (1), (2) (respectively, (1), (2), (3)) are satisfied:

(1) The divisor $D$ is étale over $S$ and coincides with the zero locus of $\chi \in \Gamma\left(X, \mathscr{L} \otimes_{\mathcal{O}_{X}} \omega_{X / S}\right)$.

(2) The global section $\chi \in \Gamma\left(X, \mathscr{L} \otimes_{\mathcal{O}_{X}} \omega_{X / S}\right)$ is a normalized Cartier eigenform associated to $(\mathscr{L}, \Theta)$.

(3) The invertible sheaf $\mathscr{L}$ is parabolically ordinary [cf. Definition A.7], i.e., the Cartier operator associated to $(\mathscr{L}, \Theta)$ is injective at every point of $S$, or, equivalently [cf. Proposition A.6], one of the following two conditions is satisfied:

- $\mathscr{L}$ is of relative order one [cf. Definition A.2], and, moreover, $X$ is parabolically ordinary $[c f$. Definition A.5, (i)].

- $\mathscr{L}$ is of relative order two [cf. Definition A.2], and, moreover, the connected finite étale double covering of $X$ which trivializes $\mathscr{L}$ [determined by $\Theta$ ] is parabolically new-ordinary [ $c f$. Definition A.5, (ii)].

Here, let us recall the following two basic questions in the p-adic Teichmüller theory discussed in [7], Introduction, \$2.1 [cf. [7], Introduction, \$2.1, (1), (2), p. 72]:

(1) Is every pointed stable curve [of type $(g, r)$, where $2 g-2+r>0$ ] hyperbolically ordinary? That is to say, does every pointed stable curve [of type $(g, r)$, where $2 g-2+r>0$ ] over $S$ admit, étale locally on $S$, a nilpotent ordinary indigenous bundle?

(2) Let $P$ be a nilpotent ordinary indigenous bundle over a pointed stable curve $X$ [of type $(g, r)$, where $2 g-2+r>0$ ] and $Y \rightarrow X$ a connected finite [log] étale covering of $X$. Then is the pull-back of $P$ to $Y$ still ordinary?

As a corollary of Theorem $\mathrm{B}$, we obtain the following theorem, which yields a negative answer to the above basic question (2) [cf. Corollary 5.4]:

THeOREM C. Let $X$ be a projective smooth curve of genus $\geq 2$ over an algebraically closed field $k$ of characteristic 3. Then, for every nilpotent ordinary indigenous bundle $P$ over $X / k$, there exists a connected finite étale covering $Y \rightarrow X$ of $X$ such that the [necessarily nilpotent admissible] indigenous bundle $(Y \rightarrow X)^{*} P$ over $Y / k$ is not ordinary. 
In $\S 6$, we give, by applying the results obtained in the present paper, a complete list of nilpotent/nilpotent admissible/nilpotent ordinary indigenous bundles over a projective smooth curve of genus two over an algebraically closed field of characteristic three [cf. Theorem 6.1]. Moreover, we prove the following theorem, which yields a partial positive answer to the above basic question (1) [cf. Corollary 6.6, Remark 6.6.1]:

THEOREM D. Every projective smooth curve of genus two over a connected noetherian scheme of characteristic three is hyperbolically ordinary [cf. [6], Chapter II, Definition 3.3, p. 1044].

\section{Construction of a dormant indigenous bundle}

In the present $\S 1$, we construct a dormant indigenous bundle over a projective smooth curve of genus $\geq 2$ of characteristic 3 [cf. Proposition 1.1 below]. In the present $\S 1$, let $g \geq 2$ be an integer, $S$ a connected noetherian scheme of characteristic 3 [i.e., over $\mathbf{F}_{3}$ ], and $f: X \rightarrow S$ a projective smooth curve [i.e., a morphism which is projective, smooth, geometrically connected, and of relative dimension one] of genus $g$ over $S$. Write $f^{F}: X^{F} \rightarrow S$ for the projective smooth curve over $S$ obtained by base-changing $X \rightarrow S$ via the absolute Frobenius morphism of $S, \Phi: X \rightarrow X^{F}$ for the relative Frobenius morphism over $S, \mathscr{I} \subseteq \mathcal{O}_{X{ }_{\times_{S}} X}$ for the ideal of $\mathcal{O}_{X \times{ }_{S} X}$ which defines the diagonal morphism with respect to $X / S$, and $X_{(n)} \subseteq X \times_{S} X$ for the closed subscheme of $X \times_{S} X$ defined by the ideal $\mathscr{I}^{n+1} \subseteq$ $\mathcal{O}_{X \times S} X$ [where $n$ is a nonnegative integer]. In particular, it follows that $\mathscr{I} / \mathscr{I}^{2}=$ $\omega_{X / S}$ (respectively, $\left.\mathscr{H}_{0 m_{\mathcal{O}_{X}}}\left(\mathscr{I} / \mathscr{I}^{2}, \mathcal{O}_{X}\right)=\tau_{X / S}\right)$, where we use the notation " $\omega$ " (respectively, " $\tau$ ") to denote the relative cotangent (respectively, tangent) sheaf.

We shall write

$$
\mathscr{B}_{\circ} \stackrel{\text { def }}{=} \operatorname{Coker}\left(\mathcal{O}_{X}{ }^{F} \rightarrow \Phi_{*} \mathcal{O}_{X}\right)
$$

for the $\mathcal{O}_{X^{F}}$-module obtained by forming the cokernel of the natural homomorphism $\mathcal{O}_{X^{F}} \rightarrow \Phi_{*} \mathcal{O}_{X}$ and

$$
\mathscr{E}_{\circ} \stackrel{\text { def }}{=} \Phi^{*} \mathscr{B}_{\circ}
$$

Since the homomorphism $\mathcal{O}_{X} \rightarrow \Phi_{*} \mathcal{O}_{X}$ admits a natural splitting after pulling back via $\Phi$, which thus determines a natural isomorphism of $\mathcal{O}_{X}$-modules

$$
\Phi^{*} \Phi_{*} \mathcal{O}_{X} \stackrel{\sim}{\rightarrow} \mathcal{O}_{X} \oplus \mathscr{E}_{\circ},
$$

and $\Phi$ is finite flat of degree 3 , it follows that $\mathscr{B}_{\circ}$, hence also $\mathscr{E}_{\circ}$, is locally free of rank 2. We shall write

$$
\pi_{\circ}: P_{\circ} \stackrel{\text { def }}{=} \mathbf{P}\left(\mathscr{E}_{\circ}\right) \rightarrow X
$$

for the $\mathbf{P}^{1}$-bundle over $X$ associated to $\mathscr{E}_{\circ}$.

Next, let us observe that one verifies immediately that the natural morphism

$$
X \times_{X^{F}} X \rightarrow X \times_{S} X
$$


determines an isomorphism

$$
X \times_{X^{F}} X \stackrel{\sim}{\rightarrow} X_{(2)}
$$

In particular, the closed immersion $X_{(1)} \hookrightarrow X \times_{S} X$ determines a closed immersion $X_{(1)} \hookrightarrow X \times_{X^{F}} X$. Thus, it follows that the $\mathcal{O}_{X}$-module $\mathscr{E}_{\circ}$, hence also the $\mathbf{P}^{1}$-bundle $P_{\circ}$, on $X$ admits a natural connection relative to $X / S$. We shall write

$$
\nabla_{\mathscr{E}_{0}}, \quad \nabla_{P_{\mathrm{o}}}
$$

for the respective natural connections on $\mathscr{E}_{\circ}, P_{\circ}$. [So one verifies immediately that the connection $\nabla_{\mathscr{E}}$ coincides with the connection on $\mathscr{E}_{\circ}=\Phi^{*} \mathscr{B} \circ$ determined by the exterior differentiation operator $\mathcal{O}_{X} \rightarrow \omega_{X / S}$.] Moreover, the above isomorphism $X \times_{X^{F}} X \stackrel{\sim}{\rightarrow} X_{(2)}$, together with the cartesian diagram

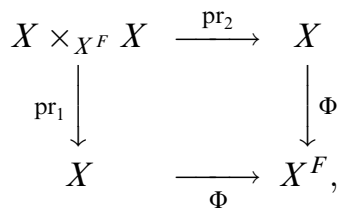

determines isomorphisms of $\mathcal{O}_{X}$-modules

$$
\Phi^{*} \Phi_{*} \mathcal{O}_{X} \stackrel{\sim}{\rightarrow} \operatorname{pr}_{1 *} \mathcal{O}_{X \times_{X} F} \stackrel{\sim}{\leftarrow} \operatorname{pr}_{1 *} \mathcal{O}_{X_{(2)}},
$$

which are compatible with the respective natural surjections onto $\mathcal{O}_{X}$ [arising from the diagonal morphism with respect to $X / X^{F}$ ] from each of these three modules. In particular, by forming the kernels of the respective natural surjections onto $\mathcal{O}_{X}$, we obtain isomorphisms of $\mathcal{O}_{X}$-modules

$$
\mathscr{E}_{\circ} \stackrel{\sim}{\rightarrow} \operatorname{Ker}\left(\operatorname{pr}_{1 *} \mathcal{O}_{X \times_{X}{ } X} \rightarrow \mathcal{O}_{X}\right) \stackrel{\sim}{\leftarrow} \operatorname{pr}_{1 *}\left(\mathscr{I} / \mathscr{I}^{3}\right) .
$$

We shall write

$$
\sigma_{\circ}: X \rightarrow P_{\circ}
$$

for the section of $\pi_{\circ}: P_{\circ} \rightarrow X$ determined by the composite $\mathscr{E}_{\circ} \rightarrow \omega_{X / S}$ of the above isomorphism $\mathscr{E}_{\circ} \stackrel{\sim}{\rightarrow} \operatorname{pr}_{1 *}\left(\mathscr{I} / \mathscr{I}^{3}\right)$ and the natural surjection $\operatorname{pr}_{1 *}\left(\mathscr{I} / \mathscr{I}^{3}\right) \rightarrow$ $\mathscr{I} / \mathscr{I}^{2}=\omega_{X / S}$. Then one verifies easily that the Kodaira-Spencer homomorphism $\sigma_{\circ}^{*} \omega_{P_{\circ} / X} \rightarrow \omega_{X / S}$ at $\sigma_{\circ}$ relative to $\nabla_{P_{\circ}}$ [i.e., the homomorphism obtained by differentiating $\sigma_{\circ}$ by means of $\nabla_{P_{\circ}}$ ] is an isomorphism. Thus, it follows immediately from our construction that the following proposition holds:

Proposition 1.1. The pair $\left(\pi_{\circ}: P_{\circ} \rightarrow X, \nabla_{P_{\circ}}\right)$ is an indigenous bundle $[c f$. Introduction] over $X / S$ whose Hodge section [cf. [6], Chapter I, Proposition 2.4, p. 1004] is given by $\sigma_{\circ}$. Moreover, the indigenous bundle $\left(\pi_{\circ}: P_{\circ} \rightarrow X, \nabla_{P_{\circ}}\right)$ is dormant [cf. Introduction].

Proof. The fact that the pair $\left(\pi_{\circ}: P_{\circ} \rightarrow X, \nabla_{P_{\circ}}\right)$ is an indigenous bundle over $X / S$ has already been verified. The fact that the indigenous bundle $\left(\pi_{\circ}: P_{\circ} \rightarrow X, \nabla_{P_{\circ}}\right)$ is dormant follows immediately from the definition of the 
connection $\nabla_{P_{\mathrm{o}}}$ [i.e., the construction of $\nabla_{P_{\mathrm{o}}}$ via the relative Frobenius morphism $\Phi]$. This completes the proof of Proposition 1.1.

In the remainder of the present $\S 1$, let us consider the invertible sheaves

$$
\operatorname{det}\left(\mathscr{E}_{\circ}\right), \quad \operatorname{det}\left(\mathscr{B}_{\circ}\right), \quad \operatorname{det}\left(\Phi_{*} \omega_{X / S}\right) .
$$

Write $\mathscr{M} \stackrel{\text { def }}{=} \mathscr{H}_{0 m_{O_{X}}}\left(\operatorname{det}\left(\mathscr{B}_{\circ}\right), \omega_{X^{F} / S}\right)$. First, let us observe that since the $\mathcal{O}_{X^{-}}$ module $\operatorname{pr}_{1 *}\left(\mathscr{I} / \mathscr{I}^{3}\right) \cong \mathscr{E}_{\circ}=\Phi^{*} \mathscr{B}_{\circ}$ fits into an exact sequence of $\mathscr{O}_{X}$-modules

$$
0 \rightarrow \omega_{X / S}^{\otimes 2} \rightarrow \operatorname{pr}_{1 *}\left(\mathscr{I} / \mathscr{I}^{3}\right) \rightarrow \omega_{X / S} \rightarrow 0,
$$

it follows that

$$
\operatorname{det}\left(\mathscr{E}_{\circ}\right) \cong \omega_{X / S}^{\otimes 3}
$$

hence also

$$
\Phi^{*} \mathscr{M} \cong \mathcal{O}_{X}
$$

Next, let us recall from the discussion preceding [9], Théorème 4.1.1, that the map

$$
\begin{aligned}
\Phi_{*} \mathcal{O}_{X} \times \Phi_{*} \mathcal{O}_{X} & \rightarrow \omega_{X} / S \\
(f, g) & \mapsto c\left(f \cdot \Phi_{*} d(g)\right)
\end{aligned}
$$

-where we write $d: \mathcal{O}_{X} \rightarrow \omega_{X / S}$ for the exterior differentiation operator and $c: \Phi_{*} \omega_{X / S} \rightarrow \omega_{X^{F} / S}$ for the Cartier operator-determines an isomorphism of $\mathcal{O}_{X}$-modules

$$
\mathscr{B}_{\circ} \stackrel{\sim}{\rightarrow} \mathscr{H}_{o m_{O_{X}}}\left(\mathscr{B}_{\circ}, \omega_{X^{F} / S}\right),
$$

which thus implies that

$$
\mathscr{M}^{\otimes 2} \cong \mathcal{O}_{X^{F}}
$$

Thus, we obtain:

\section{LEMMA 1.2. It holds that}

$$
\operatorname{det}\left(\mathscr{E}_{\circ}\right) \cong \omega_{X / S}^{\otimes 3}, \quad \operatorname{det}\left(\mathscr{B}_{\circ}\right) \cong \omega_{X^{F} / S}, \quad \operatorname{det}\left(\Phi_{*} \omega_{X / S}\right) \cong \omega_{X^{F} / S}^{\otimes 2}
$$

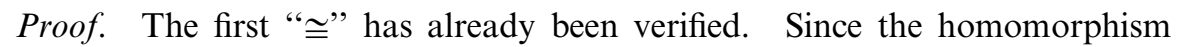
between the relative Jacobian varieties of $X^{F} / S, X / S$ induced by $\Phi$ is finite flat of degree $3^{g}$, it follows from the fact that $\Phi^{*} \mathscr{M} \cong \mathcal{O}_{X}, \mathscr{M}^{\otimes 2} \cong \mathcal{O}_{X}$ verified above that $\mathscr{M}$ lies in $\left(f^{F}\right)^{*} \operatorname{Pic}(S)$. Thus, again by the fact that $\Phi^{*} \mathscr{M} \cong \mathcal{O}_{X}$, the

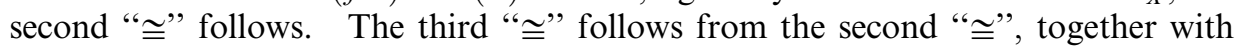
the well-known exact sequence of $\mathcal{O}_{X^{F}}$-modules

$$
0 \longrightarrow \mathcal{O}_{X^{F}} \longrightarrow \Phi_{*} \mathcal{O}_{X} \stackrel{\Phi_{*} d}{\longrightarrow} \Phi_{*} \omega_{X / S} \stackrel{c}{\longrightarrow} \omega_{X^{F} / S} \longrightarrow 0
$$

[cf., e.g., [4], Theorem 7.2]. 


\section{The dormant trivialization of the Schwarz torsor}

In the present $\S 2$, we maintain the notation of the preceding $\S 1$. In particular, we have a projective smooth curve $f: X \rightarrow S$ and a dormant indigenous bundle $\left(\pi_{\circ}: P_{\circ} \rightarrow X, \nabla_{P_{\circ}}\right)$ over $X / S$ [cf. Proposition 1.1]. We shall write

$$
\mathscr{M}_{g}
$$

for the moduli stack of projective smooth curves of genus $g$ of characteristic 3 and

$$
\mathscr{N}_{g}[\infty]
$$

for the moduli stack of projective smooth curves of genus $g$ of characteristic 3 equipped with dormant indigenous bundles. The starting point of the present $\$ 2$ is the following theorem:

THEOREM 2.1. Every dormant indigenous bundle over $X / S$ is isomorphic to the dormant indigenous bundle $\left(\pi_{\circ}: P_{\circ} \rightarrow X, \nabla_{P_{\circ}}\right)$ of Proposition 1.1.

Proof. To verify Theorem 2.1, let us first recall some facts on the p-adic Teichmüller theory [cf. [6], [7]]. The natural (1-)morphism

$$
\mathscr{N}_{g}[\infty] \rightarrow \mathscr{M}_{g}
$$

is finite and faithfully flat; moreover, there exists a dense open substack of $\mathscr{M}_{g}$ on which this (1-)morphism is étale [cf. the final portion of [7], Chapter II, Theorem 2.8, p. 153]. Thus, to complete the verification of Theorem 2.1, it suffices to verify Theorem 2.1 for a "sufficiently general" [i.e., in $\mathscr{M}_{g}$ ] projective smooth curve of genus $g$ over an algebraically closed field of characteristic three.

Next, let us observe that it follows from [11], Corollary 5.4, together with [5], Theorem 2.1, that, for every odd prime number $p$ and an integer $g \geq 2$, the number of isomorphism classes of dormant indigenous bundles over a "sufficiently general" projective smooth curve of genus $g$ over an algebraically closed field of characteristic $p$ is equal to

$$
\frac{p^{g-1}}{2^{2 g-1}} \cdot \sum_{i=1}^{p-1} \frac{1}{\sin ^{2 g-2}\left(\frac{\pi \cdot i}{p}\right)}=\frac{(-p)^{g-1}}{2} \cdot \sum_{\zeta^{p}=1, \zeta \neq 1} \frac{\zeta^{g-1}}{(\zeta-1)^{2 g-2}} .
$$

On the other hand, one verifies easily that the above quantity in the case where $p=3$ is always equal to 1 . This completes the proof of Theorem 2.1.

Remark 2.1.1. Let us observe that Theorem 2.1 also follows from the theory of molecules given in [7] [or the theory of Ehrhart quasi-polynomials discussed in [5] — cf. [5], Theorem 3.9] as follows: By considering dormant indigenous bundles over not only smooth curves but also stable curves, we have a natural extension of the (1-)morphism $\mathscr{N}_{g}[\infty] \rightarrow \mathscr{M}_{g}$ whose codomain is the moduli stack of 
stable curves of genus $g$ of characteristic 3 [i.e., " $\bar{M}_{g}$ "]. Then it follows from [7], Chapter II, Theorem 2.8, p. 153, together with a similar argument to the argument applied in the first paragraph of the proof of Theorem 2.1, that, to complete the verification of Theorem 2.1 , it suffices to verify that

a structure of dormant molecule [cf. [7], Chapter V, §0, p. 229] on a fixed [nonpointed] totally degenerate stable curve of characteristic 3 is unique.

On the other hand, this follows immediately from [7], Introduction, Theorem 1.3, pp. 41-42, together with the fact that $\#\left(\left(\mathbf{F}_{3} /\{ \pm 1\}\right) \backslash\{0\}\right)=1$.

Remark 2.1.2. One may also replace the second paragraph of the proof of Theorem 2.1 by the local computation of the p-curvature given in the discussion preceding Proposition 3.1 below [cf. Remark 3.1.1 below].

It follows from Theorem 2.1 [together with the discussion given in the first paragraph of proof of Theorem 2.1] that the natural (1-)morphism

$$
\mathscr{N}_{g}[\infty] \rightarrow \mathscr{M}_{g}
$$

is an isomorphism, hence also étale. Thus, by the final portion of [11], Theorem 3.3, we obtain:

COROllary 2.2. Every dormant indigenous bundle over $X / S$ is dormant ordinary [cf. [11], Definition 3.2].

We shall write

$$
\mathscr{C}_{g} \rightarrow \mathscr{M}_{g}
$$

for the universal curve over $\mathscr{M}_{g}$ and

$$
\mathscr{S}_{g} \rightarrow \mathscr{M}_{g}
$$

for the Schwarz torsor over $\mathscr{M}_{g}$ [cf. [7], Introduction, $\$ 0.4$, pp. 7-9], i.e., the torsor over the locally free coherent $\mathcal{O}_{\mathcal{M}_{g}}$-module of rank $3 g-3$

$$
\left(\mathscr{C}_{g} \rightarrow \mathscr{M}_{g}\right)_{*} \omega_{\mathscr{C}_{g} / \mathscr{M}_{g}}^{\otimes 2}
$$

obtained by forming the moduli stack of projective smooth curves of genus $g$ of characteristic 3 equipped with indigenous bundles [cf. also [6], Chapter I, Corollary 2.9, p. 1007]. By considering the composite of the above natural isomorphism $\mathscr{M}_{g} \leftarrow \mathscr{N}_{g}[\infty]$ and the natural closed immersion $\mathscr{N}_{g}[\infty] \hookrightarrow \mathscr{S}_{g}$ of stacks, we obtain a trivialization

$$
\mathscr{M}_{g} \rightarrow \mathscr{S}_{g}
$$

of the Schwarz torsor. 
Definition 2.3. We shall refer to this trivialization $\mathscr{M}_{g} \rightarrow \mathscr{S}_{g}$ of the Schwarz torsor as the dormant trivialization.

By the dormant trivialization of Definition 2.3, we obtain an isomorphism of $\mathscr{S}_{g}$ with the geometric vector bundle over $\mathscr{M}_{g}$ associated to $\left(\mathscr{C}_{g} \rightarrow \mathscr{M}_{g}\right)_{*} \omega_{\mathscr{C}_{g} / \mathscr{M}_{g}}^{\otimes 2}$. Thus:

COROLlary 2.4. There exists a natural bijection between the following two sets:

- $\Gamma\left(S, f_{*} \omega_{X / S}^{\otimes 2}\right)=\Gamma\left(X, \omega_{X / S}^{\otimes 2}\right)$.

- The set of isomorphism classes of indigenous bundles over $X / S$.

For $\theta \in \Gamma\left(S, f_{*} \omega_{X / S}^{\otimes 2}\right)=\Gamma\left(X, \omega_{X / S}^{\otimes 2}\right)$, the indigenous bundle over $X / S$ corresponding to $\theta$ is given as follows: Let us recall the pair $\left(\mathscr{E}_{0}, \nabla_{\mathscr{E}_{0}}\right)$ and the exact sequence of $\mathcal{O}_{X}$-modules

$$
0 \rightarrow \omega_{X / S}^{\otimes 2} \rightarrow \mathscr{E}_{\circ} \rightarrow \omega_{X / S} \rightarrow 0
$$

discussed in $\S 1$. Write $\phi^{\theta}: \mathscr{E}_{\circ} \rightarrow \mathscr{E}_{\circ} \otimes_{\mathcal{O}_{X}} \omega_{X / S}$ for the homomorphism of $\mathcal{O}_{X^{-}}$ modules obtained by forming the composite

$$
\mathscr{E}_{\circ} \rightarrow \omega_{X / S} \stackrel{\theta}{\rightarrow} \omega_{X / S}^{\otimes 3}=\omega_{X / S}^{\otimes 2} \otimes_{\mathcal{O}_{X}} \omega_{X / S} \hookrightarrow \mathscr{E}_{\circ} \otimes_{\mathcal{O}_{X}} \omega_{X / S}
$$

We shall write

$$
\nabla_{P \text { 。 }}^{\theta}
$$

for the connection on $P_{\circ}$ determined by the connection

$$
\nabla_{\mathscr{E}_{\circ}}^{\theta} \stackrel{\text { def }}{=} \nabla_{\mathscr{E}_{\circ}}+\phi^{\theta}
$$

on $\mathscr{E}_{0} . \quad$ Then the indigenous bundle over $X / S$ corresponding to $\theta$ is given by

$$
P_{\theta} \stackrel{\text { def }}{=}\left(\pi_{\circ}: P_{\circ} \rightarrow X, \nabla_{P_{\circ}}^{\theta}\right) .
$$

Moreover, for $\theta \in \Gamma\left(S, f_{*} \omega_{X / S}^{\otimes 2}\right)=\Gamma\left(X, \omega_{X / S}^{\otimes 2}\right)$, the dormant locus in $S$ of $P_{\theta}$ [i.e., the maximal closed subscheme $F \subseteq S$ of $S$ such that the restriction of $P_{\theta}$ to $X \times{ }_{S} F$ is dormant] coincides with the zero locus in $S$ of $\theta[i . e .$, the maximal closed subscheme $F \subseteq S$ of $S$ such that the restriction of $\theta$ to $X \times_{S} F$ is identically zero].

Remark 2.4.1. We note that $\operatorname{since} \operatorname{det}\left(\mathscr{E}_{0}\right) \cong \omega_{X / S}^{\otimes 3} \not \mathcal{O}_{X}$ [cf. Lemma 1.2], the pair $\left(\mathscr{E}_{\circ}, \nabla_{\mathscr{E}_{\circ}}\right)$, as well as the pair $\left(\mathscr{E}_{\circ}, \nabla_{\mathscr{E}_{\circ}}^{\theta}\right)$ [cf. Corollary 2.4], is not an indigenous vector bundle [cf. [6], Chapter I, Definition 2.2, pp. 1002-1003; also the discussion preceding [6], Chapter I, Definition 2.2, p. 1002]. One verifies easily from the fact that $\operatorname{det}\left(\mathscr{B}_{\circ}\right) \cong \omega_{X^{F} / S}$ [cf. Lemma 1.2] that if $\mathscr{L}$ is an invertible sheaf on $X^{F}$ such that $\mathscr{L}^{\otimes 2} \cong \tau_{X^{F} / S}$ [note that since 2 is invertible on $S$, such an invertible sheaf always exists after étale localizing $S]$, then an indigenous vector bundle whose projectivization is isomorphic to $\left(\pi_{\circ}: P_{\circ} \rightarrow X, \nabla_{P_{\circ}}\right)$ is given by 
tensoring $\left(\mathscr{E}_{0}, \nabla_{\mathscr{E}_{0}}\right)$ with the invertible sheaf $\Phi^{*} \mathscr{L}$ equipped with the connection determined by the exterior differentiation operator $\mathcal{O}_{X} \rightarrow \omega_{X / S}$. On the other hand, one also verifies easily that the operation of taking tensor product with a dormant invertible sheaf [i.e., an invertible sheaf equipped with a connection whose $p$-curvature is identically zero] does not affect the local computation of the p-curvature as given in the discussion preceding Proposition 3.1 below.

\section{Local criteria}

In the present $\$ 3$, we prove local criteria for some properties on indigenous bundles [cf. Proposition 3.1; Proposition 3.8, (ii), below]. We maintain the notation introduced at the beginning of $\S 1$.

Let

$$
\theta \in \Gamma\left(X, \omega_{X / S}^{\otimes 2}\right)
$$

be a global section of $\omega_{X / S}^{\otimes 2}$. Thus, it follows from Corollary 2.4 that we obtain a connection

$$
\nabla_{P \text { 。 }}^{\theta}
$$

on the $\mathbf{P}^{1}$-bundle $P_{\circ}$ such that the pair

$$
P_{\theta} \stackrel{\text { def }}{=}\left(\pi_{\circ}: P_{\circ} \rightarrow X, \nabla_{P_{\circ}}^{\theta}\right)
$$

forms an indigenous bundle over $X / S$.

Let $x \in X$ be a point of $X$ and $t_{x}=t \in \mathcal{O}_{X}$ a local parameter of $X / S$ at $x$. Write $\phi_{x}=\phi \in \mathcal{O}_{X}$ for the local function on $X$ at $x$ which fits into the equality

$$
\theta=\phi \cdot d t \otimes d t
$$

Then one verifies immediately that the local sections

$$
e_{1} \stackrel{\text { def }}{=} 1 \otimes t-t \otimes 1, \quad e_{2} \stackrel{\text { def }}{=} e_{1}^{2} \in \operatorname{pr}_{1 *} \mathcal{O}_{X \times_{X}{ }^{F}} \stackrel{\sim}{\leftarrow} \Phi^{*} \Phi_{*} \mathcal{O}_{X}
$$

[cf. the discussion preceding Proposition 1.1] are contained in the submodules

$$
\operatorname{Ker}\left(\operatorname{pr}_{1 *} \mathcal{O}_{X \times_{X} F} \rightarrow \mathcal{O}_{X}\right) \stackrel{\sim}{\leftarrow} \mathscr{E}_{0},
$$

and that, in the natural exact sequence of $\mathcal{O}_{X}$-modules

$$
0 \rightarrow \omega_{X / S}^{\otimes 2} \rightarrow \mathscr{E}_{\circ} \rightarrow \omega_{X / S} \rightarrow 0
$$

the local section $e_{2}$ determines a local trivialization of the invertible sheaf $\omega_{X / S}^{\otimes 2}$, and the local section $e_{1}$ determines a local splitting of the surjection $\mathscr{E}_{\circ} \rightarrow \omega_{X / S}$; in particular, $\left\{e_{1}, e_{2}\right\}$ forms a local basis of $\mathscr{E}_{\circ}$.

Next, let us observe that it follows immediately from the definition of $\nabla_{\mathscr{E}}$ that

$$
\nabla_{\mathscr{E}_{0}}\left(e_{1}, e_{2}\right)=\left(e_{1}, e_{2}\right) \cdot\left(\begin{array}{ll}
0 & 1 \\
0 & 0
\end{array}\right) \otimes d t
$$


Thus, one verifies immediately from the definition of $\nabla_{\mathscr{E}_{\circ}}^{\theta}[\mathrm{cf}$. Corollary 2.4] that

$$
\nabla_{\mathscr{E}_{0}}^{\theta}\left(e_{1}, e_{2}\right)=\left(e_{1}, e_{2}\right) \cdot\left(\begin{array}{ll}
0 & 1 \\
\phi & 0
\end{array}\right) \otimes d t
$$

In particular, it follows that the $p$-curvature $\mathscr{P}^{\theta}$ of the connection $\nabla_{\mathscr{E}_{\circ}}^{\theta}$ [cf., e.g., the discussion preceding [4], Theorem 5.1] is given by

$$
\begin{aligned}
\mathscr{P}^{\theta}: \Phi^{*} \tau_{X^{F} / S} & \rightarrow \mathscr{A} d_{\mathscr{O}_{X}}\left(\mathscr{E}_{\circ}\right) \\
\Phi^{-1} \delta_{t^{F}} & \mapsto\left(\left(e_{1}, e_{2}\right) \mapsto\left(e_{1}, e_{2}\right) \cdot\left(\begin{array}{cc}
-\phi^{\prime} & \phi \\
\phi^{2}+\phi^{\prime \prime} & \phi^{\prime}
\end{array}\right)\right)
\end{aligned}
$$

-where we write $t^{F} \in \mathcal{O}_{X^{F}}$ for the local parameter of $X^{F} / S$ determined by the local parameter $t \in \mathcal{O}_{X}, \delta_{t^{F}}$ (respectively, $\delta_{t}$ ) for the local trivialization of $\tau_{X^{F} / S}$ (respectively, $\tau_{X / S}$ ) which maps $d t^{F}$ (respectively, $d t$ ) to $1, \partial_{t}$ for the local derivation corresponding to $\delta_{t}$, " $(-)^{\prime \prime}$ " for " $\partial_{t}(-)$ " [i.e., " $(-)^{\prime \prime}$ " is the "derivative of $(-)$ with respect to $\left.t^{\prime \prime}\right]$, and

$$
\mathscr{A} d_{\mathcal{O}_{X}}\left(\mathscr{E}_{\circ}\right) \subseteq \mathscr{E} n d_{\Theta_{X}}\left(\mathscr{E}_{\circ}\right)
$$

for the submodule of $\mathscr{E} n d_{\mathcal{O}_{X}}\left(\mathscr{E}_{\circ}\right)$ consisting of trace zero endomorphisms of locally free coherent $\mathscr{O}_{X}$-module $\mathscr{E}_{\circ}$. This local computation [cf. Remark 2.4.1] leads us to the following local criteria for some properties on indigenous bundles:

Proposition 3.1. The following hold:

(i) The indigenous bundle $P_{\theta}$ is nilpotent [cf. Introduction] if and only if, for every point $x \in X$, the equality

$$
\left(\phi_{x}^{\prime}\right)^{2}+\phi_{x} \cdot \phi_{x}^{\prime \prime}+\phi_{x}^{3}=0
$$

holds.

(ii) Suppose that $S$ is the spectrum of an algebraically closed field [of characteristic 3]. Then the indigenous bundle $P_{\theta}$ is admissible [cf. Introduction] if and only if, for every closed point $x \in X$, it holds that

$$
\operatorname{ord}_{x}\left(\phi_{x}\right) \leq 2 \text {. }
$$

Proof. Assertion (i) follows from the definition, together with the above local computation. To verify assertion (ii), let us observe that

$$
\left\{\left(\begin{array}{ll}
0 & 1 \\
0 & 0
\end{array}\right), \quad\left(\begin{array}{cc}
1 & 0 \\
0 & -1
\end{array}\right), \quad\left(\begin{array}{ll}
0 & 0 \\
1 & 0
\end{array}\right)\right\}
$$

forms a local basis of the locally free coherent $\mathcal{O}_{X}$-module $\mathscr{A} d_{\mathcal{O}_{X}}\left(\mathscr{E}_{\circ}\right)$. Thus, assertion (ii) follows immediately from the definition, together with the above local computation.

Remark 3.1.1. If $\mathscr{P}^{\theta}=0$, then it follows from the above local computation that $\phi=0$, hence also $\theta=0$. By means of this observation, one can give an alternative proof of Theorem 2.1 [cf. Remark 2.1.2]. 
Next, let us observe that the natural exact sequence of $\mathcal{O}_{X}$-modules

$$
0 \rightarrow \omega_{X / S}^{\otimes 2} \rightarrow \mathscr{E}_{\circ} \rightarrow \omega_{X / S} \rightarrow 0
$$

determines a homomorphism of $\mathcal{O}_{X}$-modules

$$
\mathscr{A} d_{\mathcal{O}_{X}}\left(\mathscr{E}_{0}\right) \hookrightarrow \mathscr{E} n d_{\mathcal{O}_{X}}\left(\mathscr{E}_{0}\right) \rightarrow \mathscr{H}_{o m_{\mathcal{O}_{X}}}\left(\omega_{X / S}^{\otimes 2}, \omega_{X / S}\right) \cong \tau_{X} / S
$$

moreover, the square Hasse invariant [cf. Introduction] of the indigenous bundle $P_{\theta}$ is defined as the composite of the $p$-curvature $\mathscr{P}^{\theta}$ and this homomorphism. Thus, by the above local computation, we obtain:

Proposition 3.2. The square Hasse invariant of the indigenous bundle $P_{\theta}$ is, up to multiplication by a global section of $\mathcal{O}_{S}^{\times}$, given by

$$
\theta \in \Gamma\left(X, \omega_{X / S}^{\otimes 2}\right) \cong \Gamma\left(X, \mathscr{H}_{0 m_{\mathcal{O}_{X}}}\left(\Phi^{*} \tau_{X^{F} / S}, \tau_{X / S}\right)\right) .
$$

In particular, if, moreover, the indigenous bundle $P_{\theta}$ is admissible, then the double supersingular divisor [cf. Introduction] of $P_{\theta}$ coincides with the zero locus of $\theta$.

In particular, we obtain the following two corollaries:

Corollary 3.3. Suppose that the indigenous bundle $P_{\theta}$ is nilpotent and admissible. Then the supersingular divisor [cf. Introduction] of $P_{\theta}$ is finite étale over $S$.

Proof. Since [it follows from the definition that] the supersingular divisor of $P_{\theta}$ is finite flat over $S$ [cf. also [6], Chapter II, Proposition 2.6, (2), p. 1032], to complete the verification of Corollary 3.3 , it suffices to verify the unramifiedness. Thus, we may assume without loss of generality that $S$ is the spectrum of an algebraically closed field [of characteristic 3]. Then the unramifiedness follows from Proposition 3.1, (ii); Proposition 3.2, together with the definition of the supersingular divisor.

COROLlary 3.4. Suppose that $S$ is reduced. Then the isomorphism class of nilpotent indigenous bundle over $X / S$ is completely determined by the zero locus of the square Hasse invariant.

Proof. First, let us observe that since $S$ is reduced, it follows from [7], Chapter I, Proposition 1.5, p. 91, that, to verify Corollary 3.4, we may assume without loss of generality that $S$ is the spectrum of an algebraically closed field $k$ [of characteristic 3]. Next, let us observe that one verifies easily that if $\phi$ is nonzero and satisfies the equality " $\left(\phi^{\prime}\right)^{2}+\phi \cdot \phi^{\prime \prime}+\phi^{3}=0$ " of Proposition 3.1, (i), then, for every $c \in k \backslash\{0,1\}, c \cdot \phi$ does not satisfy the equality " $\left(\phi^{\prime}\right)^{2}+\phi \cdot \phi^{\prime \prime}+\phi^{3}$ $=0$ " of Proposition 3.1, (i). Thus, Corollary 3.4 follows from Proposition 3.1, (i); Proposition 3.2, together with Corollary 2.4. 
Remark 3.4.1. Observe that Corollary 3.4 is a generalization of [6], Chapter II, Proposition 2.6, (4), p. 1032, in the case where $p=3$.

Next, let us observe that it follows from the equality of Proposition 3.1, (i), that the following lemma holds:

Lemma 3.5. Suppose that $S$ is the spectrum of an algebraically closed field [of characteristic 3], and that the indigenous bundle $P_{\theta}$ is nilpotent. Then, for every closed point $x \in X$, it holds that $\operatorname{ord}_{x}\left(\phi_{x}\right) \notin 3 \mathbf{Z}+1$. Write

Proof. Assume that $n \stackrel{\text { def }}{=} \operatorname{ord}_{x}\left(\phi_{x}\right) \in 3 \mathbf{Z}+1$ for some closed point $x \in X$.

$$
\phi_{x}=\sum_{i=0}^{\infty} a_{i} t_{x}^{i}
$$

by regarding $\phi_{x}$ as an element of the completion $\mathcal{O}_{X, x}^{\wedge}$. Then, by considering the coefficient of the " $t_{x}^{2 n-2}$ " of the left-hand side of the equality " $\left(\phi^{\prime}\right)^{2}+\phi \cdot \phi^{\prime \prime}+\phi^{3}$ $=0$ " of Proposition 3.1, (i), we obtain that $a_{n}=0$. Thus, we obtain a contradiction.

By Lemma 3.5, we obtain:

COROLlary 3.6. Suppose that $g=2$. If a nilpotent indigenous bundle over $X / S$ is active [cf. [7], Chapter II, Definition 1.1, p. 127], then it is admissible.

Proof. Let us first observe that it follows from the definition of admissibility that, to verify Corollary 3.6, we may assume without loss of generality that $S$ is the spectrum of an algebraically closed field $k$ [of characteristic 3]. On the other hand, in this case, since $\operatorname{deg}\left(\omega_{X / S}^{\otimes 2}\right)=4$, it follows immediately from Proposition 3.1, (ii), together with Lemma 3.5, that every nilpotent and active indigenous bundle over $X / S$ is admissible.

We shall write

$$
\mathscr{N}_{g}
$$

for the moduli stack of smooth nilcurves [cf. the discussion preceding [7], Introduction, Theorem 0.1, p. 24] of genus $g$ of characteristic 3, i.e., the moduli stack of projective smooth curves of genus $g$ of characteristic 3 equipped with nilpotent indigenous bundles. Note that it follows from [6], Chapter II, Theorem 2.3, p. 1029 [cf. also the discussion following [6], Chapter II, Definition 2.4, p. 1030], that the natural (1-)morphism

$$
\mathscr{N}_{g} \rightarrow \mathscr{M}_{g}
$$

is finite flat of degree $3^{3 g-3}$. 
Corollary 3.7. Suppose that $g=2$. Then the open substack of $\mathscr{N}_{2}$

$$
\mathscr{N}_{2} \backslash \mathscr{N}_{2}[\infty]
$$

is smooth over $\mathbf{F}_{3}$.

Proof. This follows from Corollary 3.6, together with [6], Chapter II, Corollary 2.16, p. 1043.

Proposition 3.8. Suppose that $S$ is the spectrum of an algebraically closed field $k$ [of characteristic 3], and that the indigenous bundle $P_{\theta}$ is nilpotent. Then the following hold:

(i) We shall write

$$
T_{\theta}
$$

for the relative tangent space of $\mathscr{N}_{g} / \mathscr{M}_{g}$ at the $k$-valued point of $\mathscr{N}_{g}$ corresponding to $P_{\theta}$. Then $T_{\theta}$ is naturally isomorphic to the subspace of $\Gamma\left(X, \omega_{X / S}^{\otimes 2}\right)$ consisting of global sections $\eta$ of $\omega_{X / S}^{\otimes 2}$ such that if, for some closed point $x \in X$, we write

$$
\eta=\psi_{x} \cdot d t_{x} \otimes d t_{x}
$$

then it holds that

$$
\left(\phi_{x} \cdot \psi_{x}\right)^{\prime \prime}=0 .
$$

(ii) It holds that the indigenous bundle $P_{\theta}$ is ordinary [cf. Introduction] if and only if the following condition is satisfied: For every nonzero global section $\eta$ of $\omega_{X / S}^{\otimes 2}$, if, for some closed point $x \in X$, we write

$$
\eta=\psi_{x} \cdot d t_{x} \otimes d t_{x}
$$

then it holds that

$$
\left(\phi_{x} \cdot \psi_{x}\right)^{\prime \prime} \neq 0
$$

Proof. Assertion (ii) follows immediately from assertion (i). Thus, to complete the verification of Proposition 3.8, it suffices to verify assertion (i). Write $A \stackrel{\text { def }}{=} k[\varepsilon] /\left(\varepsilon^{2}\right)$, where $\varepsilon$ is an indeterminate. Then it follows from Proposition 3.1, (i), that $T_{\theta}$ is naturally isomorphic to the subspace of $\Gamma\left(X, \omega_{X / S}^{\otimes 2}\right)$ consisting of global sections $\eta$ of $\omega_{X / S}^{\otimes 2}$ such that if, for some closed point $x \in X$, we write

$$
\eta=\psi_{x} \cdot d t_{x} \otimes d t_{x}
$$

then the equality

$$
\left((\phi+\varepsilon \psi)^{\prime}\right)^{2}+(\phi+\varepsilon \psi) \cdot(\phi+\varepsilon \psi)^{\prime \prime}+(\phi+\varepsilon \psi)^{3}=0
$$

-where write $\psi \stackrel{\text { def }}{=} \psi_{x}$-in $A \otimes_{k} \Gamma\left(X, \omega_{X / S}^{\otimes 2}\right)=\Gamma\left(X, \omega_{X / S}^{\otimes 2}\right) \oplus \varepsilon \cdot \Gamma\left(X, \omega_{X / S}^{\otimes 2}\right)$ holds. On the other hand, again by Proposition 3.1, (i), one verifies easily that it holds that this equality holds if and only if the equality

$$
\phi^{\prime \prime} \cdot \psi+\phi \cdot \psi^{\prime \prime}-\phi^{\prime} \cdot \psi^{\prime}\left(=(\phi \cdot \psi)^{\prime \prime}\right)=0
$$

holds. This completes the proof of assertion (i). 
NILPOTENT ADMISSIBLE INDIGENOUS BUNDLES IN CHARACTERISTIC THREE 705

Remark 3.8.1. Proposition 3.8, (ii), also follows immediately from Proposition 3.2; Lemma A.9, (i) [in the case where we take the pair " $(\mathscr{L}, \Theta)$ " of Lemma A.9, (i), to be the pair consisting of $\mathcal{O}_{X}$ and the natural identification $\mathcal{O}_{X} \otimes_{\mathcal{O}_{X}} \mathcal{O}_{X}=\mathcal{O}_{X}-\mathrm{cf}$. Remark A.4.1], together with [6], Chapter II, Proposition 2.12, p. 1037.

Thus, we obtain:

Corollary 3.9. Suppose that $S$ is the spectrum of an algebraically closed field $k$ [of characteristic 3], and that the indigenous bundle $P_{\theta}$ is nilpotent. Then the following conditions are equivalent:

(1) The indigenous bundle $P_{\theta}$ is dormant.

(2) The vector space $T_{\theta}$ over $k$ of Proposition 3.8, (i), is of dimension $\mathbf{3 g} \mathbf{- 3}$.

Proof. If $P_{\theta}$ is dormant, then $\theta=0$ [cf. Corollary 2.4]. Thus, the implication $(1) \Rightarrow(2)$ follows from Proposition 3.8, (i). On the other hand, if condition (2) is satisfied, then it follows from Proposition 3.8, (i) [in the case where we take the " $\eta$ " of Proposition 3.8, (i), to be $\theta]$, that $\left(\phi^{2}\right)^{\prime \prime}=0$. Thus, since $0=\left(\phi^{2}\right)^{\prime \prime}=-\left(\phi^{\prime}\right)^{2}-\phi \cdot \phi^{\prime \prime}=\phi^{3}$ [cf. Proposition 3.1, (i)], we conclude that $\phi=0$, hence also $\theta=0$, i.e., that condition (1) is satisfied [cf. Corollary 2.4]. This completes the proof of Corollary 3.9.

\section{Indigenous bundles arising from squares}

In the present $\S 4$, we discuss some properties on an indigenous bundle which arises from the square of a "twisted" differential form, i.e., the square of a global section of a "square root" of the square of the relative cotangent sheaf [cf. Proposition 4.1, Proposition 4.2, Proposition 4.4 below]. In the present $\S 4$, we maintain the notation introduced at the beginning of $\S 1$.

Let

$$
\mathscr{L}=\left(\mathscr{L}, \Theta: \mathscr{L}^{\otimes 2} \stackrel{\sim}{\rightarrow} \mathcal{O}_{X}\right)
$$

be a square-trivialized invertible sheaf on $X$ [cf. Definition A.3] and

$$
\chi \in \Gamma\left(X, \mathscr{L} \otimes_{\mathcal{O}_{X}} \omega_{X / S}\right)
$$

a global section of $\mathscr{L} \otimes_{\mathcal{O}_{X}} \omega_{X / S}$. Let us recall [cf. the discussion following Definition A.3] that we have isomorphisms of invertible sheaves

$$
\begin{gathered}
\mathscr{L} \stackrel{\sim}{\rightarrow} \mathscr{L}^{\otimes 3} \stackrel{\sim}{\rightarrow} \Phi^{*} \mathscr{L}^{F} \\
\Theta(l \otimes l) \cdot l \mapsto l \otimes l \otimes l \mapsto \Phi^{-1} l^{F}
\end{gathered}
$$

- where we write $\mathscr{L}^{F}$ for the invertible sheaf on $X^{F}$ obtained by pulling back $\mathscr{L}$ via the morphism $X^{F} \rightarrow X$ induced by the absolute Frobenius morphism of $S, l$ is a local section of $\mathscr{L}$, and $l^{F}$ is the local section of $\mathscr{L}^{F}$ determined by $l$. 
Let $x \in X$ be a point of $X, t_{x}=t \in \mathcal{O}_{X}$ a local parameter of $X / S$ at $x$, and $l_{x}=l \in \mathscr{L}$ a local trivialization of $\mathscr{L}$ at $x$. Then the global trivialization $\Theta$ and the local trivialization $l_{x}=l$ determine a local unit

$$
\delta_{x}=\delta \stackrel{\text { def }}{=} \Theta(l \otimes l) \in \mathcal{O}_{X}^{\times}
$$

at $x$. Moreover, the global section $\chi$ determines a local function $\phi_{x}=\phi \in \mathcal{O}_{X}$ on $X$ at $x$ which fits into the equality

$$
\chi=\phi \cdot l \otimes d t
$$

at $x$.

Next, let us observe that the trivialization $\Theta$ determines an isomorphism

$$
\Theta: \Gamma\left(X,\left(\mathscr{L} \otimes_{\mathcal{O}_{X}} \omega_{X / S}\right)^{\otimes 2}\right) \stackrel{\sim}{\rightarrow} \Gamma\left(X, \omega_{X / S}^{\otimes 2}\right) .
$$

Thus, by considering the image via this isomorphism of the square

$$
\theta \stackrel{\text { def }}{=} \chi \otimes \chi \in \Gamma\left(X,\left(\mathscr{L} \otimes_{\mathcal{O}_{X}} \omega_{X / S}\right)^{\otimes 2}\right)
$$

of $\chi$, we obtain a global section

$$
\Theta(\theta) \in \Gamma\left(X, \omega_{X / S}^{\otimes 2}\right)
$$

of $\omega_{X / S}^{\otimes 2}$. On the other hand, it follows from Corollary 2.4 that this global section $\Theta(\theta)$ gives rise to an indigenous bundle over $X / S$

$$
P_{\Theta(\theta)} \stackrel{\text { def }}{=}\left(\pi_{\circ}: P_{\circ} \rightarrow X, \nabla_{P_{\circ}}^{\Theta(\theta)}\right) .
$$

Proposition 4.1. Suppose that $\chi$ defines a relative effective Cartier divisor of $X / S$. Then the following conditions are equivalent:

(1) The indigenous bundle $P_{\Theta(\theta)}$ is nilpotent and active.

(2) The global section $\chi \in \Gamma\left(X, \mathscr{L} \otimes_{\mathcal{O}_{X}} \omega_{X / S}\right)$ is a normalized Cartier eigenform associated to $\mathscr{L}=(\mathscr{L}, \Theta)[c f$. Definition A.8, (i)].

Proof. Let us first observe that it follows from the definitions of $\Theta(\theta)$ that $\Theta(\theta)$ fits into the equality

$$
\Theta(\theta)=\phi^{2} \cdot \delta \cdot d t \otimes d t
$$

at $x$. Thus, it follows from Proposition 3.1, (i), that it holds that $P_{\Theta(\theta)}$ is nilpotent if and only if, for every point $x \in X$,

$$
\begin{aligned}
\left(\left(\phi^{2}\right.\right. & \left.\cdot \delta)^{\prime}\right)^{2}+\left(\phi^{2} \cdot \delta\right) \cdot\left(\phi^{2} \cdot \delta\right)^{\prime \prime}+\left(\phi^{2} \cdot \delta\right)^{3} \\
& =\phi^{4} \cdot\left(\delta^{\prime}\right)^{2}-\phi^{3} \cdot \phi^{\prime} \cdot \delta \cdot \delta^{\prime}-\phi^{3} \cdot \phi^{\prime \prime} \cdot \delta^{2}+\phi^{4} \cdot \delta \cdot \delta^{\prime \prime}+\phi^{6} \cdot \delta^{3} \\
& =\phi^{3} \cdot \delta^{3} \cdot\left(-\left(\phi \cdot \delta^{-1}\right)^{\prime \prime}+\phi^{3}\right)
\end{aligned}
$$

is equal to zero. In particular, Proposition 4.1 follows from Lemma A.9, (ii), together with Corollary 2.4. 
Proposition 4.2. Suppose that the indigenous bundle $P_{\Theta(\theta)}$ is nilpotent and active. Then the following conditions are equivalent:

(1) The indigenous bundle $P_{\Theta(\theta)}$ is nilpotent and admissible.

(2) The zero locus of the global section $\chi \in \Gamma\left(X, \mathscr{L} \otimes_{\mathcal{O}_{X}} \omega_{X / S}\right)$ is finite étale over $S$.

Proof. Since [one verifies immediately that] the locus [in $S$ ] on which condition (1) (respectively, (2)) is satisfied is open, to complete the verification of Proposition 4.2, we may assume without loss of generality that $S$ is the spectrum of an algebraically closed field [of characteristic 3]. Then the equivalence $(1) \Leftrightarrow(2)$ follows from Proposition 3.1, (ii), together with the definition of $\Theta(\theta)$.

Remark 4.2.1.

(i) Note that condition (2) of Proposition 4.1 does not imply condition (2) of Proposition 4.2. Such a counter-example is as follows: Let $k$ be an algebraically closed field of characteristic 3. Let us consider the following polynomial:

$$
f(t)=t^{12}+t^{10}+1 \in k[t] .
$$

Then one verifies easily that $f(t)$ does not have any multiple root, which thus implies that the equation

$$
s^{2}=f(t)
$$

determines a hyperelliptic projective smooth curve $C$ of genus five over $k$.

Write $\omega \in \Gamma\left(C, \omega_{C / k}\right)$ for the global section of $\omega_{C / k}$ whose restriction to the open subscheme of $X$ on which $f$ is invertible is of the form

$$
\frac{\alpha \cdot t^{4}}{s} d t
$$

-where $\alpha \in k$ satisfies that $\alpha^{2}=2$. Then one verifies easily from Lemma A.9, (ii), that $\omega$ is a normalized Cartier eigenform associated to $\mathcal{O}_{C}$ [equipped with the natural identification $\left.\mathcal{O}_{C} \otimes_{\mathcal{O}_{C}} \mathcal{O}_{C}=\mathcal{O}_{C}\right]$. On the other hand, it is immediate that if we write $c \in C$ for the closed point corresponding to $(t, s)=(0,1)$, then $\operatorname{ord}_{c}(\omega)=4$.

(ii) It follows from Corollary 3.6 that a nilpotent active indigenous bundle over a projective smooth curve of genus two in characteristic three is admissible. On the other hand, it follows from the discussion of (i), together with Proposition 4.1 and Proposition 4.2, that there exists a nilpotent active indigenous bundle over a projective smooth curve in characteristic three which is not admissible.

Proposition 4.3. Suppose that $S$ is the spectrum of an algebraically closed field $k$ [of characteristic 3], and that the indigenous bundle $P_{\Theta(\theta)}$ is nilpotent and admissible. Write

$$
T_{\Theta(\theta)}
$$


for the relative tangent space of $\mathscr{N}_{g} / \mathscr{M}_{g}$ at the $k$-valued point of $\mathscr{N}_{g}$ corresponding to $P_{\Theta(\theta)}$. Thus, it follows from Proposition 3.8, (i), that $T_{\Theta(\theta)}$ may be regarded as a subspace of $\Gamma\left(X, \omega_{X / S}^{\otimes 2}\right)$ :

$$
T_{\Theta(\theta)} \subseteq \Gamma\left(X, \omega_{X / S}^{\otimes 2}\right)
$$

Then the map

$$
\begin{aligned}
\Gamma\left(X, \mathscr{L} \otimes_{\mathcal{O}_{X}} \omega_{X / S}\right) \otimes_{k} \Gamma\left(X, \mathscr{L} \otimes_{\mathcal{O}_{X}} \omega_{X / S}\right) & \rightarrow \Gamma\left(X, \omega_{X / S}^{\otimes 2}\right) \\
\alpha \otimes \beta & \mapsto \Theta(\alpha \otimes \beta)
\end{aligned}
$$

induces an isomorphism of vector spaces over $k$

$$
\begin{aligned}
\operatorname{Ker}\left(C_{\mathscr{L}}\right) & \stackrel{\sim}{\rightarrow} T_{\Theta(\theta)} \\
\sigma & \mapsto \Theta(\sigma \otimes \chi)
\end{aligned}
$$

-where we write $C_{\mathscr{L}}$ for the Cartier operator associated to $\mathscr{L}=(\mathscr{L}, \Theta)$ [cf. Definition A.4].

Proof. Let us first observe that [one verifies easily that] the homomorphism of vector spaces over $k$

$$
\begin{aligned}
\Xi: \Gamma\left(X, \mathscr{L} \otimes_{\mathcal{O}_{X}} \omega_{X / S}\right) & \rightarrow \Gamma\left(X, \omega_{X / S}^{\otimes 2}\right) \\
\alpha & \mapsto \Theta(\alpha \otimes \chi)
\end{aligned}
$$

is injective. Thus, to verify Proposition 4.3, it suffices to verify the following two assertions:

(a) $\Xi\left(\operatorname{Ker}\left(C_{\mathscr{L}}\right)\right) \subseteq T_{\Theta(\theta)}$.

(b) The resulting [cf. (a)] homomorphism $\Xi: \operatorname{Ker}\left(C_{\mathscr{L}}\right) \rightarrow T_{\Theta(\theta)}$ is surjective. Next, let us recall from the proof of Proposition 4.1 that $\Theta(\theta)$ fits into the equality

$$
\Theta(\theta)=\phi^{2} \cdot \delta \cdot d t \otimes d t
$$

at $x$. Thus, it follows from Proposition 3.8, (i), that the subspace $T_{\Theta(\theta)} \subseteq$ $\Gamma\left(X, \omega_{X / S}^{\otimes 2}\right)$ consists of global sections $\eta$ of $\omega_{X / S}^{\otimes 2}$ such that if, for some closed point $x \in X$, we write

$$
\eta=\psi \cdot d t \otimes d t
$$

then it holds that

$$
\left(\phi^{2} \cdot \delta \cdot \psi\right)^{\prime \prime}=0
$$

Now we verify the assertion (a). Let $\sigma \in \Gamma\left(X, \mathscr{L} \otimes_{\mathcal{O}_{X}} \omega_{X / S}\right)$ be such that $C_{\mathscr{L}}(\sigma)=0$. Write

$$
\sigma=\mu \cdot l \otimes d t
$$


at $x$. Since $\sigma \in \operatorname{Ker}\left(C_{\mathscr{L}}\right)$, it holds that $\left(\mu \cdot \delta^{-1}\right)^{\prime \prime}=0$ [cf. Lemma A.9, (i)]. Thus, since

$$
\Theta(\sigma \otimes \chi)=\phi \cdot \mu \cdot \delta \cdot d t \otimes d t
$$

at $x$, and

$\left(\phi^{3} \cdot \mu \cdot \delta^{2}\right)^{\prime \prime}=\left(\phi^{3} \cdot \delta^{3}\right)^{\prime \prime} \cdot\left(\mu \cdot \delta^{-1}\right)-\left(\phi^{3} \cdot \delta^{3}\right)^{\prime} \cdot\left(\mu \cdot \delta^{-1}\right)^{\prime}+\left(\phi^{3} \cdot \delta^{3}\right) \cdot\left(\mu \cdot \delta^{-1}\right)^{\prime \prime}=0$,

we conclude that $\Xi(\sigma) \in T_{\Theta(\theta)}$. This completes the proof of the assertion (a).

Next, we verify the assertion (b). Let $\eta$ be a global section of $\omega_{X / S}^{\otimes 2}$ which belongs to $T_{\Theta(\theta)}$. Write

$$
\eta=\psi \cdot d t \otimes d t
$$

at $x$. Then since

$$
\begin{aligned}
0= & \left(\phi^{2} \cdot \delta \cdot \psi\right)^{\prime \prime} \\
= & \left(-\left(\phi^{\prime}\right)^{2}-\phi \cdot \phi^{\prime \prime}\right) \cdot \delta \cdot \psi+\phi^{2} \cdot \delta^{\prime \prime} \cdot \psi+\phi^{2} \cdot \delta \cdot \psi^{\prime \prime} \\
& +\phi \cdot \phi^{\prime} \cdot \delta^{\prime} \cdot \psi-\phi^{2} \cdot \delta^{\prime} \cdot \psi^{\prime}+\phi \cdot \phi^{\prime} \cdot \delta \cdot \psi^{\prime},
\end{aligned}
$$

and $\phi$ is of order $\leq 1$ [at $x$ ] by Proposition 4.2, it holds that $\operatorname{ord}_{x}(\phi) \geq 1$ implies $\operatorname{ord}_{x}(\psi) \geq 1$. Thus, it follows that $V(\chi)=V(\chi)_{\text {red }} \subseteq V(\eta)_{\text {red }} \subseteq V(\eta)$, where we write " $V(-)$ " for the zero locus of " $(-)$ ", i.e., that $\eta \in \Gamma\left(X, \omega_{X / S}^{\otimes 2}(-V(\chi))\right) \subseteq$ $\Gamma\left(X, \omega_{X / S}^{\otimes 2}\right)$. Now let us observe that since $\left(\mathscr{L} \otimes_{\mathcal{O}_{X}} \omega_{X / S}\right)^{\otimes 2} \cong \omega_{X / S}^{\otimes 2}$, which thus implies that $\mathscr{L} \otimes_{\mathcal{O}_{X}} \omega_{X / S} \cong \omega_{X / S}^{\otimes 2}(-V(\chi))$, we have an isomorphism

$$
\begin{aligned}
\Gamma\left(X, \mathscr{L} \otimes_{\mathcal{O}_{X}} \omega_{X / S}\right) & \stackrel{\sim}{\rightarrow} \Gamma\left(X, \omega_{X / S}^{\otimes 2}(-V(\chi))\right) \\
\sigma & \mapsto \Theta(\sigma \otimes \chi) .
\end{aligned}
$$

Thus, we conclude that there exists a global section $\sigma$ of $\mathscr{L} \otimes_{\mathcal{O}_{X}} \omega_{X / S}$ such that $\eta=\Theta(\sigma \otimes \chi)$. Write

$$
\sigma=\mu \cdot l \otimes d t
$$

at $x$, which thus implies that

$$
\psi=\mu \cdot \phi \cdot \delta
$$

at $x$. Then since

$$
\begin{aligned}
0 & =\left(\phi^{2} \cdot \delta \cdot \psi\right)^{\prime \prime}=\left(\mu \cdot \phi^{3} \cdot \delta^{2}\right)^{\prime \prime} \\
& =\left(\phi^{3} \cdot \delta^{3}\right)^{\prime \prime} \cdot\left(\mu \cdot \delta^{-1}\right)-\left(\phi^{3} \cdot \delta^{3}\right)^{\prime} \cdot\left(\mu \cdot \delta^{-1}\right)^{\prime}+\left(\phi^{3} \cdot \delta^{3}\right) \cdot\left(\mu \cdot \delta^{-1}\right)^{\prime \prime} \\
& =\phi^{3} \cdot \delta^{3} \cdot\left(\mu \cdot \delta^{-1}\right)^{\prime \prime},
\end{aligned}
$$

it holds that $\left(\mu \cdot \delta^{-1}\right)^{\prime \prime}=0$, i.e., that $\sigma \in \operatorname{Ker}\left(C_{\mathscr{L}}\right)$ [cf. Lemma A.9, (i)]. This completes the proof of the assertion (b), hence also of Proposition 4.3. 
Proposition 4.4. Suppose that the indigenous bundle $P_{\Theta(\theta)}$ is nilpotent and admissible. Then the following conditions are equivalent:

(1) The indigenous bundle $P_{\Theta(\theta)}$ is nilpotent and ordinary.

(2) The invertible sheaf $\mathscr{L}$ is parabolically ordinary [cf. Definition A.7].

Proof. Since [one verifies immediately that the locus [in $S$ ] on which condition (1) (respectively, (2)) is satisfied is open, to complete the verification of Proposition 4.4, we may assume without loss of generality that $S$ is the spectrum of an algebraically closed field [of characteristic 3]. Then Proposition 4.4 follows from Proposition 4.3.

\section{Nilpotent admissible indigenous bundles via Cartier operators}

In the present $\S 5$, we prove the main result of the present paper [cf. Theorem 5.2 below], as well as some corollaries to the main result. In the present $\S 5$, we maintain the notation introduced at the beginning of $\S 1$.

DEFINITION 5.1.

(i) We shall say that a pair

$$
\left(\mathscr{L}, \chi \in \Gamma\left(X, \mathscr{L} \otimes_{\mathcal{O}_{X}} \omega_{X / S}\right)\right)
$$

consisting of an invertible sheaf $\mathscr{L}$ on $X$ and a global section $\chi$ of $\mathscr{L}_{\mathcal{O}_{X}} \omega_{X / S}$ is of CE-type [where "CE" stands for "Cartier Eigenform"] if

- $\mathscr{L}^{\otimes 2} \cong \mathcal{O}_{X}$,

- $\chi \in \Gamma\left(X, \mathscr{L} \otimes_{\mathcal{O}_{X}} \omega_{X / S}\right)$ is a Cartier eigenform associated to $\mathscr{L}$ [cf. Definition A.8, (ii)], and

- the zero locus of $\chi$ is étale over $S$.

(ii) We shall say that a pair $(\mathscr{L}, \chi)$ of CE-type is of CEO-type [where "CEO" stands for "Cartier Eigenform and Ordinary"] if $\mathscr{L}$ is parabolically ordinary.

(iii) We shall say that a relative effective Cartier divisor $D$ of $X / S$ is of $C E$-type (respectively, of CEO-type) if there exists a pair $(\mathscr{L}, \chi)$ of CE-type (respectively, of CEO-type) such that $D$ coincides with the zero locus of $\chi$.

The main result of the present paper is as follows:

THEOREM 5.2. Let $g \geq 2$ be an integer, $S$ a connected noetherian scheme of characteristic 3 [i.e., over $\mathbf{F}_{3}$ ], and $f: X \rightarrow S$ a projective smooth curve of genus $g$. Write $\omega_{X / S}$ for the relative cotangent bundle of $X / S$. Then the following hold:

(i) Let $P$ be a nilpotent admissible indigenous bundle over $X / S$. Write $\mathscr{L}_{P}$ for the Hasse defect of $P\left[c f\right.$. Definition B.2] and $\chi_{P} \in \Gamma\left(X, \mathscr{L}_{P} \otimes_{\mathcal{O}_{X}} \omega_{X / S}\right)$ for the Hasse invariant of $P$ [cf. also the final portion of Proposition B.4]. Then the pair

$$
\left(\mathscr{L}_{P}, \chi_{P}\right)
$$

is of CE-type [cf. Definition 5.1, (i)]. Moreover, it holds that $P$ is nilpotent ordinary if and only if the pair $\left(\mathscr{L}_{P}, \chi_{P}\right)$ is of CEO-type [cf. Definition 5.1, (ii)]. 
(ii) Let $D$ be a relative effective Cartier divisor of $X / S$. Then it holds that $D$ is the supersingular divisor of a nilpotent admissible (respectively, nilpotent ordinary) indigenous bundle over $X / S$ if and only if $D$ is of CE-type (respectively, of CEO-type) [ $c f$. Definition 5.1, (iii)].

(iii) Suppose that $S$ is reduced. Then, by considering the supersingular divisors, we have a bijection between the following two sets:

- The set of isomorphism classes of nilpotent admissible (respectively, nilpotent ordinary) indigenous bundles over $X / S$.

- The set of relative effective Cartier divisors of $X / S$ of CE-type (respectively, of CEO-type).

Proof. First, we verify the first assertion of assertion (i). Let us first observe that it follows from the final portion of Proposition B.3 that $\mathscr{L}_{P}^{\otimes 2} \cong \mathcal{O}_{X}$. Moreover, it follows from Corollary 3.3 that the zero locus of $\chi_{P}$ is finite étale over $S$. Thus, to complete the verification of the first assertion of assertion (i), it suffices to verify that there exists a trivialization $\Theta: \mathscr{L}_{P}^{\otimes 2} \stackrel{\sim}{\rightarrow} \mathcal{O}_{X}$ such that $\chi_{P}$ is a normalized Cartier eigenform associated to $\left(\mathscr{L}_{P}, \Theta\right)$.

Let us write $\theta_{P} \in \Gamma\left(X, \omega_{X / S}^{\otimes 2}\right)$ for the global section of $\omega_{X / S}^{\otimes 2}$ corresponding, via the bijection of Corollary 2.4, to the indigenous bundle $P$. Fix a trivialization $\Theta: \mathscr{L}_{P}^{\otimes 2} \stackrel{\sim}{\rightarrow} \mathcal{O}_{X}$ of $\mathscr{L}_{P}^{\otimes 2}$ and write $\theta$ for the image via the isomorphism $\Gamma\left(X,\left(\mathscr{L}_{P} \otimes_{\mathcal{O}_{X}} \omega_{X / S}\right)^{\otimes 2}\right) \stackrel{\sim}{\rightarrow} \Gamma\left(X, \omega_{X / S}^{\otimes 2}\right)$ induced by $\Theta$ of the square $\chi_{P} \otimes \chi_{P} \in$ $\Gamma\left(X,\left(\mathscr{L}_{P} \otimes_{\mathcal{O}_{X}} \omega_{X / S}\right)^{\otimes 2}\right)$ of $\chi_{P}$. Then it follows from Proposition 3.2 that there exists a global unit $u \in \Gamma\left(S, \mathcal{O}_{S}^{\times}\right)$such that $\theta_{P}=u \cdot \theta$. Thus, we may assume without loss of generality, by replacing $\Theta$ by $u^{-1} \cdot \Theta$, that $\theta_{P}=\theta$. In particular, it follows from Proposition 4.1 that $\chi_{P}$ is a normalized Cartier eigenform associated to $\left(\mathscr{L}_{P}, \Theta\right)$. This completes the proof of the first assertion of assertion (i). Moreover, the final assertion of assertion (i) follows from the first assertion of assertion (i), together with Proposition 4.4 [cf. also the equality " $\theta_{P}=\theta$ " in the proof of the first assertion of assertion (i)]. This completes the proof of assertion (i).

Next, we verify assertion (ii). The necessity follows from assertion (i). To verify the sufficiency, let $D$ be a relative effective Cartier divisor of $X / S$ of CE-type (respectively, of CEO-type). Thus, it follows from the definition that there exists a pair $(\mathscr{L}, \chi)$ of CE-type (respectively, of CEO-type) such that $D$ is defined by $\chi$. Now since $(\mathscr{L}, \chi)$ is of CE-type, the zero locus of $\chi$ is étale over $S$, and there exists a trivialization $\Theta: \mathscr{L}^{\otimes 2} \stackrel{\sim}{\rightarrow} \mathcal{O}_{X}$ of $\mathscr{L}^{\otimes 2}$ such that $\chi$ is a normalized Cartier eigenform associated to $(\mathscr{L}, \Theta)$. Thus, it follows from Proposition 4.1 and Proposition 4.2 that the indigenous bundle $P$ over $X / S$ corresponding, via the bijection of Corollary 2.4 , to the image via the isomorphism $\Gamma\left(X,\left(\mathscr{L} \otimes_{\mathcal{O}_{X}} \omega_{X / S}\right){ }^{\otimes 2}\right) \stackrel{\sim}{\rightarrow} \Gamma\left(X, \omega_{X / S}^{\otimes 2}\right)$ induced by $\Theta$ of the square $\chi \otimes \chi \in \Gamma\left(X,\left(\mathscr{L} \otimes_{\mathcal{O}_{X}} \omega_{X / S}\right)^{\otimes 2}\right)$ of $\chi$ is nilpotent and admissible. Moreover, it follows from Proposition 4.4 that if $(\mathscr{L}, \chi)$ is of CEO-type, then the indigenous bundle $P$ is ordinary. Write $\chi_{P}$ for the Hasse invariant of $P$. Then it follows from Proposition 3.2 that the zero locus of $\chi_{P}$, i.e., the supersingular divisor of 
$P$, coincides with the zero locus of $\chi$, i.e., $D$. This completes the proof of the sufficiency, hence also of assertion (ii).

The injectivity of the map of assertion (iii) follows from Corollary 3.4 [cf. also [6], Chapter II, Proposition 2.6, (4), p. 1032]. The surjectivity of the map of assertion (iii) follows from assertion (ii). This completes the proof of Theorem 5.2.

COROLlaRY 5.3. Let $X$ be a projective smooth curve of genus $g \geq 2$ over an algebraically closed field $k$ of characteristic 3 and $\mathscr{L}$ an invertible sheaf on $X$ such that $\mathscr{L}^{\otimes 2} \cong \mathcal{O}_{X}$. Then the following hold:

(i) Suppose that $\mathscr{L} \cong \mathcal{O}_{X}$ (respectively, $\mathscr{L} \not \mathcal{O}_{X}$ ). Then the number of isomorphism classes of nilpotent admissible indigenous bundles over $X / k$ whose Hasse defects are isomorphic to $\mathscr{L}$ is

$$
\leq \# \mathbf{P}^{g-1}\left(\mathbf{F}_{3}\right)=\frac{3^{g}-1}{3-1} \quad\left(\text { respectively }, \leq \# \mathbf{P}^{g-2}\left(\mathbf{F}_{3}\right)=\frac{3^{g-1}-1}{3-1}\right) .
$$

(ii) The number of isomorphism classes of nilpotent admissible indigenous bundles over $X / k$ is

$$
\begin{aligned}
& \leq \# \mathbf{P}^{g-1}\left(\mathbf{F}_{3}\right)+\left(\#\left((\mathbf{Z} / 2 \mathbf{Z})^{\oplus 2 g}\right)-1\right) \cdot \# \mathbf{P}^{g-2}\left(\mathbf{F}_{3}\right) \\
& =\frac{1}{2}\left(\left(3^{g}-1\right)+\left(2^{2 g}-1\right)\left(3^{g-1}-1\right)\right) .
\end{aligned}
$$

Proof. First, we verify assertion (i). Fix a trivialization $\Theta: \mathscr{L}^{\otimes 2} \stackrel{\sim}{\rightarrow} \mathcal{O}_{X}$. Let us first observe that it follows from Theorem 5.2, (iii) [cf. also Remark A.8.1], that, to verify assertion (i), it suffices to verify that the number of subspaces of $V_{\mathscr{L}} \stackrel{\text { def }}{=} \Gamma\left(X, \mathscr{L} \otimes_{\mathcal{O}_{X}} \omega_{X / S}\right)$ of dimension 1 which are preserved and not annihilated by the Cartier operator $\left.V_{\mathscr{L}} \rightarrow \Gamma\left(X^{F}, \mathscr{L}^{F} \otimes_{\mathcal{O}_{X} F} \omega_{X^{F} / S}\right)\right) \stackrel{\sim}{\leftarrow} V_{\mathscr{L}}$ associated to $(\mathscr{L}, \Theta)$ is

$$
\leq \# \mathbf{P}^{g-1}\left(\mathbf{F}_{3}\right)=\frac{3^{g}-1}{3-1} \quad\left(\text { respectively }, \leq \# \mathbf{P}^{g-2}\left(\mathbf{F}_{3}\right)=\frac{3^{g-1}-1}{3-1}\right)
$$

if $\mathscr{L} \cong \mathcal{O}_{X}$ (respectively, $\mathscr{L} \not \mathcal{O}_{X}$ ). To this end, let us observe that one verifies easily from the Riemann-Roch theorem that the vector space $V_{\mathscr{L}}$ over $k$ is of dimension $g$ (respectively, $g-1$ ) if $\mathscr{L} \cong \mathcal{O}_{X}$ (respectively, $\mathscr{L} \not \mathcal{O}_{X}$ ). Thus, assertion (i) follows immediately from elementary linear algebra [cf. also [8], Corollary, p. 143]. This completes the proof of assertion (i). Assertion (ii) follows immediately from assertion (i). This completes the proof of Corollary 5.3.

COROllary 5.4. Let $X$ be a projective smooth curve of genus $\geq 2$ over an algebraically closed field $k$ of characteristic 3. Then, for every nilpotent ordinary indigenous bundle $P$ over $X / k$, there exists a connected finite étale covering $Y \rightarrow X$ 
of $X$ such that the [necessarily nilpotent admissible] indigenous bundle $(Y \rightarrow X)^{*} P$ over $Y / k$ is not ordinary.

Proof. Write $\mathscr{L}_{P}$ for the Hasse defect of $P$ and $Y_{1} \rightarrow X$ for the connected finite étale covering of $X$ which trivializes $\mathscr{L}_{P}$. [So if $\mathscr{L}_{P} \cong \mathcal{O}_{X}$ (respectively, $\mathscr{L}_{P} \not \mathcal{O}_{X}$ ), then $Y_{1} \rightarrow X$ is of degree 1 (respectively, 2).] Next, let $Y_{2} \rightarrow X$ be a connected finite étale covering of $X$ such that $Y_{2}$ is not parabolically ordinary [cf., e.g., [10], Théorème 2] and $Y \rightarrow X$ a connected finite étale covering of $X$ which dominates $Y_{1} \rightarrow X$ and $Y_{2} \rightarrow X$.

Now let us observe that since $Y \rightarrow X$ factors through $Y_{1} \rightarrow X$, one verifies immediately that the Hasse defect of the indigenous bundle $(Y \rightarrow X)^{*} P$ over $Y / k$ is trivial. Thus, it follows from the final portion of Theorem 5.2, (i), that the indigenous bundle $(Y \rightarrow X)^{*} P$ over $Y / k$ is ordinary if and only if $Y$ is parabolically ordinary [cf. also Proposition A.6]. On the other hand, since $Y \rightarrow X$ factors through $Y_{2} \rightarrow X$, and $Y_{2}$ is not parabolically ordinary, it holds that $Y$ is not parabolically ordinary [cf., e.g., the discussion entitled "The $p$-rank" of [8], pp. 146-147 and [8], Corollary 1, p. 174], which thus implies that the indigenous bundle $(Y \rightarrow X)^{*} P$ over $Y / k$ is not ordinary.

Remark 5.4.1. Corollary 5.4 yields a negative answer to the basic question (2) of [7], Introduction, §2.1, p. 72.

Finally, we discuss the various moduli stacks related to the main result of the present paper. We shall apply the notational conventions for the various stacks established in the Appendix C [in the case where we take the " $p$ " of the Appendix $\mathrm{C}$ to be 3]. The following corollary follows immediately from the final portion of Theorem 5.2, (i) [cf. also Proposition C.5]:

COROllary 5.5. We have a cartesian diagram of stacks

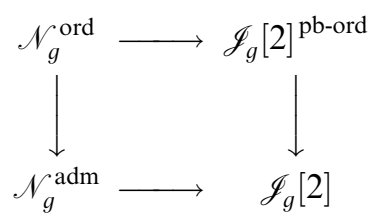

- where the vertical arrows are the natural open immersions of stacks [cf. Definition C.4], and the lower horizontal arrow is the Hasse defect morphism [cf. Definition C.1].

Next, for a nonnegative integer $r$, write

$$
\mathscr{M}_{g,[r]}
$$

for the moduli stack of hyperbolic curves of type $(g, r)$ of characteristic 3, i.e., the moduli stack of projective smooth curves of genus $g$ of characteristic 3 equipped with relative effective étale Cartier divisors of relative degree $r$. 
Definition 5.6. It follows from Corollary 3.3, together with Proposition B.3, that the supersingular divisor of the universal nilpotent admissible indigenous bundle over $\mathscr{C}_{g} \times \mathscr{M}_{g} \mathscr{N}_{g}^{\mathrm{adm}} \rightarrow \mathscr{N}_{g}^{\mathrm{adm}}$ determines a (1-)morphism over $\mathscr{M}_{g}$

$$
\mathscr{N}_{g}^{\mathrm{adm}} \rightarrow \mathscr{M}_{g,[2 g-2]}
$$

We shall refer to this (1-)morphism as the Hasse morphism.

The following corollary follows immediately from Theorem 5.2, (ii):

Corollary 5.7. Let $(X, D)$ be a hyperbolic curve of type $(g, 2 g-2)$ over a connected noetherian scheme $S$ of characteristic 3 . Then the following conditions are equivalent:

(1) The classifying (1-)morphism $S \rightarrow \mathscr{M}_{g,[2 g-2]}$ of $(X, D)$ factors through the Hasse morphism $\mathscr{N}_{g}^{\text {adm }} \rightarrow \mathscr{M}_{g,[2 g-2]}$.

(2) The relative effective Cartier divisor $D$ of $X / S$ is of CE-type.

\section{The case of genus two}

In the present $\S 6$, we give, by applying the results obtained in the present paper, a complete list of nilpotent/nilpotent admissible/nilpotent ordinary indigenous bundles over a projective smooth curve of genus two over an algebraically closed field of characteristic three [cf. Theorem 6.1 below]. Moreover, we also prove that every projective smooth curve of genus two over a connected noetherian scheme of characteristic three is hyperbolically ordinary [cf. Corollary 6.6 below]. In the present $\S 6$, we maintain the notation introduced at the beginning of $\S 1$. Suppose, moreover, that $g=2$ [i.e., that $X$ is of genus 2], and that $S$ is the spectrum of an algebraically closed field $k$ [of characteristic 3].

Since $X$ is of genus 2, X admits a uniquely determined hyperelliptic involution $l$, which determines a double covering

$$
\xi: X \rightarrow Q
$$

-where we write $Q$ for the [scheme-theoretic] quotient of $X$ by the action of $\imath$. [Thus, $Q$ is isomorphic to the projective line $\mathbf{P}_{k}^{1}$ over $k$.] We shall write

$$
\mathrm{WP} \subseteq X
$$

for the ramification locus of $\xi$, i.e., the zero locus of the global section of the invertible sheaf on $X$ [of degree 6]

$$
\mathscr{H} \mathrm{m}_{\mathcal{O}_{X}}\left(\xi^{*} \omega_{Q / S}, \omega_{X / S}\right)
$$

determined by $\xi$. [Thus, $\mathrm{WP} \subseteq X$ is the set of Weierstrass points of $X$.] Then, as is well-known, the closed subscheme WP $\subseteq X$ is reduced; moreover, we have a bijection [of finite sets of cardinality 15] between

and

- the set of subsets of WP of cardinality 2

- the set of isomorphism classes of invertible sheaves on $X$ of order 2 
given by mapping $D=\left\{x_{1}, x_{2}\right\} \subseteq \mathrm{WP}$ to $\mathscr{L}_{D} \stackrel{\text { def }}{=} \mathcal{O}_{X}\left(x_{1}-x_{2}\right)$. Finally, for a subset $D \subseteq \mathrm{WP}$ of cardinality 2 , write

$$
\xi_{D}: X_{D} \rightarrow X
$$

for the connected finite étale double covering which trivializes $\mathscr{L}_{D}$ and

$$
E_{D}(\rightarrow Q)
$$

for the elliptic curve over $k$ obtained by considering the double covering of $Q$ $\left(\cong \mathbf{P}_{k}^{1}\right)$ whose branch locus coincides with $\xi(\mathrm{WP} \backslash D)$. Then one verifies immediately from the definition of $E_{D}$ that we obtain a cartesian diagram

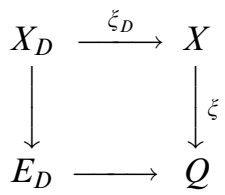

which thus implies that the "new part" of $\xi_{D}$ [i.e., the abelian variety obtained by forming the quotient of the Jacobian variety of $X_{D}$ by the image-via the homomorphism induced by $\xi_{D}$ - of the Jacobian variety of $\left.X\right]$ is isogenous to $E_{D}$.

By this observation, together with the results obtained in the present paper, we give the following complete list of nilpotent/nilpotent admissible/nilpotent ordinary indigenous bundles over $X / S$ :

THEOREM 6.1. The following hold:

(i) Every nilpotent nondormant indigenous bundle over $X / S$ is admissible.

(ii) Let $D \subseteq \mathrm{WP}$ be a subset of cardinality 2 and $\theta_{D} \in \Gamma\left(X, \omega_{X / S}^{\otimes 2}\right)$ a [uniquely determined, up to multiplication by an element of $\left.k^{\times}\right]$global section of $\omega_{X / S}^{\otimes 2}$ such that the zero locus of $\theta_{D}$ coincides with $2 D$ [if we naturally regard $D$ as a reduced divisor of degree 2], and, moreover, the elliptic curve $E_{D}$ is ordinary. Then $a$ [uniquely determined-cf. Proposition 3.2, Corollary 3.4] $k^{\times}$-multiple of $\theta_{D}$ corresponds, via the bijection of Corollary 2.4, to a nilpotent [necessarily admissible - c $f$. (i)] indigenous bundle over $X / S$.

(iii) Let $\omega_{\mathrm{CE}} \in \Gamma\left(X, \omega_{X / S}\right)$ be a Cartier eigenform associated to $\mathcal{O}_{X}$. Then a [uniquely determined-cf. Proposition 3.2, Corollary 3.4] $k^{\times}$-multiple of $\omega_{\mathrm{CE}} \otimes \omega_{\mathrm{CE}}$ $\in \Gamma\left(X, \omega_{X / S}^{\otimes 2}\right)$ corresponds, via the bijection Corollary 2.4, to a nilpotent [necessarily admissible $-c f$. (i)] indigenous bundle over $X / S$.

(iv) Every nilpotent nondormant [i.e., admissible - $c f$. (i)] indigenous bundle over $X / S$ is obtained as the result of either (ii) or (iii).

(v) It holds that a nilpotent indigenous bundle over $X / S$ is ordinary if and only if one of the following two conditions is satisfied:

(1) The indigenous bundle is obtained as the result of (ii).

(2) The indigenous bundle is obtained as the result of (iii), and, moreover, $X$ is parabolically ordinary. 
Proof. Assertion (i) follows from Corollary 3.6. Next, we verify assertion (ii). Let $D \subseteq \mathrm{WP}$ be as in assertion (ii). Then it is immediate that $\mathscr{L}_{D} \otimes_{\mathcal{O}_{X}}$ $\omega_{X / S} \cong \mathcal{O}_{X}(D)$ [if we naturally regard $D$ as a reduced divisor of degree 2]. In particular, it follows that $\Gamma\left(X, \mathscr{L}_{D} \otimes_{\mathcal{O}_{X}} \omega_{X / S}\right)$ is of dimension 1, which thus implies that the zero locus of every nonzero global section of $\mathscr{L}_{D} \otimes_{\mathcal{O}_{X}} \omega_{X / S}$ coincides with [the reduced closed subscheme of $X$ whose underlying subset is] $D$. Moreover, since $E_{D}$ is ordinary, it follows from Proposition A.6 [cf. also Remark A.8.1] that every nonzero global section of $\mathscr{L}_{D} \otimes_{\mathcal{O}_{X}} \omega_{X / S}$ is a Cartier eigenform associated to $\mathscr{L}_{D}$. Thus, it follows immediately from Proposition 4.1 that assertion (ii) holds. This completes the proof of assertion (ii).

Assertion (iii) follows from Proposition 4.1. Next, we verify assertion (iv). Let $P$ be a nilpotent admissible indigenous bundle over $X / S$. If the Hasse defect of $P$ is trivial, then it follows from Theorem 5.2, (iii), that $P$ is obtained as the result of (iii). If the Hasse defect of $P$ is nontrivial, then it follows from Theorem 5.2, (iii), together with Proposition A.6, that $P$ is obtained as the result of (ii) [cf. also the proof of assertion (ii)]. This completes the proof of assertion (iv). Assertion (v) follows from the final portion of Theorem 5.2, (i), together with assertion (iv). This completes the proof of Theorem 6.1.

Remark 6.1.1. It follows immediately from Proposition 3.2, together with the various definitions involved, that the Hasse invariants and the supersingular divisors of nilpotent admissible indigenous bundles over $X / S$ are given as follows:

- Write $P$ for the nilpotent admissible indigenous bundle over $X / S$ obtained as the result of Theorem 6.1, (ii), with respect to a subset $D \subseteq \mathrm{WP}$ as in Theorem 6.1, (ii). Then the supersingular divisor of $P$ is [the reduced closed subscheme of $X$ whose underlying subset is] $D$. Next, let us observe that $\mathcal{O}_{X}(D)^{\otimes 2} \cong \omega_{X / S}^{\otimes 2}$, and, moreover, the vector space $\Gamma\left(X, \mathcal{O}_{X}(D)\right)$ over $k$ is of dimension 1 . Let $s$ be a nonzero global section of $\mathcal{O}_{X}(D)$. Then the Hasse invariant of the indigenous bundle $P$ is a $k^{\times}$-multiple of

$$
s \in \Gamma\left(X, \mathcal{O}_{X}(D)\right) .
$$

- Write $P$ for the nilpotent admissible indigenous bundle over $X / S$ obtained as the result of Theorem 6.1, (iii), with respect to a global section $\omega_{\mathrm{CE}} \in \Gamma\left(X, \omega_{X / S}\right)$ as in Theorem 6.1, (iii). Then the Hasse invariant of $P$ is a $k^{\times}$-multiple of

$$
\omega_{\mathrm{CE}} \in \Gamma\left(X, \omega_{X / S}\right) .
$$

The supersingular divisor of this indigenous bundle is the zero locus of $\omega_{\mathrm{CE}}$.

Remark 6.1.2. One verifies immediately that an indigenous bundle [implicitly] discussed in [1], $§ 11$, is a nilpotent admissible indigenous bundle obtained as the result of Theorem 6.1, (iii) [cf. the discussion in Remark 6.1.1 concerning supersingular divisors; also condition (2) of Theorem 6.1, (v), and the equivalence (a) $\Leftrightarrow(\mathrm{b})$ of [1], Theorem 2.8, (3)]. 
The following corollary follows immediately from Theorem 2.1 and Theorem 6.1 [cf. also Remark 6.1.1], together with elementary linear algebra [cf. also [8], Corollary, p. 143]:

COROLlary 6.2. Write $n_{\mathrm{WP}}$ for the number of subsets $D$ of WP of cardinality 2 such that the elliptic curve $E_{D}$ is ordinary. Write, moreover, $\gamma_{X}(\in\{0,1,2\})$ for the p-rank of the Jacobian variety of $X$. Then the following hold:

(i) The number of isomorphism classes of nilpotent indigenous bundles over $X / S$ is given by

$$
1+n_{\mathrm{WP}}+\# \mathbf{P}^{\gamma_{X}-1}\left(\mathbf{F}_{3}\right)
$$

-where we write $\# \mathbf{P}^{-1}\left(\mathbf{F}_{3}\right) \stackrel{\text { def }}{=} 0$.

(ii) The number of isomorphism classes of nilpotent admissible indigenous bundles over $X / S$ is given by

$$
n_{\mathrm{WP}}+\# \mathbf{P}^{\gamma_{X}-1}\left(\mathbf{F}_{3}\right)
$$

-where we write $\# \mathbf{P}^{-1}\left(\mathbf{F}_{3}\right) \stackrel{\text { def }}{=} 0$.

(iii) If $X$ is parabolically ordinary (respectively, not parabolically ordinary) [i.e., $\gamma_{X}=2$ (respectively, $\gamma_{X} \neq 2$ )], then the number of isomorphism classes of nilpotent ordinary indigenous bundles over $X / S$ is given by

$$
\left.n_{\mathrm{WP}}+4 \text { (respectively, } n_{\mathrm{WP}}\right) \text {. }
$$

Next, let us recall the following well-known lemma on the $p$-rank of the Jacobian variety of a projective smooth curve of genus $\leq 2$ over an algebraically closed field of characteristic three. The following well-known lemma follows immediately from, for instance, the characterization of the Cartier operator [cf., e.g., [4], Theorem 7.2], together with a well-known explicit description of the global differential forms on a hyperelliptic projective smooth curve:

LEMMA 6.3. The following hold:

(i) Suppose that $X$ admits a dense open subscheme which is isomorphic to the affine scheme over $k$

$$
\operatorname{Spec}\left(k[s, t] /\left(s^{2}-f(t)\right)\right)
$$

-where $s$ and $t$ are indeterminates, and

$$
f(t)=t^{5}+a_{4} t^{4}+a_{3} t^{3}+a_{2} t^{2}+a_{1} t+a_{0} \in k[t] .
$$

Then it holds that $X$ is parabolically ordinary if and only if $a_{1} \neq a_{2} \cdot a_{4}$.

(ii) If an elliptic curve $E$ over $k$ admits a dense open subscheme which is isomorphic to the affine scheme over $k$

$$
\operatorname{Spec}\left(k[s, t] /\left(s^{2}-f(t)\right)\right)
$$


-where $s$ and $t$ are indeterminates, and

$$
\begin{gathered}
f(t)=t^{4}+a_{3} t^{3}+a_{2} t^{2}+a_{1} t+a_{0} \in k[t] \\
\text { (respectively, } \left.f(t)=t^{3}+a_{2} t^{2}+a_{1} t+a_{0} \in k[t]\right)
\end{gathered}
$$

then it holds that $E$ is ordinary if and only if $a_{2} \neq 0$.

The following corollary was already proved in [7] [cf. Remark 6.4.1 below]:

COROllary 6.4. There exists a dense open substack of $\mathscr{M}_{2}$ such that every projective smooth curve parametrized by a geometric point on this open substack admits exactly 19 isomorphism classes of nilpotent ordinary indigenous bundles.

Proof. It follows from Theorem 6.1 [cf. also Corollary 6.2, (iii)] that, to verify Corollary 6.4, it suffices to verify the following assertion: There exists a dense open substack $U_{\text {ord }} \subseteq \mathscr{M}_{2}$ (respectively, $U_{\mathrm{WP}} \subseteq \mathscr{M}_{2}$ ) of $\mathscr{M}_{2}$ such that every projective smooth curve parametrized by a geometric point on $U_{\text {ord }}$ (respectively, $U_{\mathrm{WP}}$ ) is parabolically ordinary (respectively, satisfies the condition that, for every subset $D \subseteq \mathrm{WP}$ of cardinality 2 , the elliptic curve $E_{D}$ is ordinary). On the other hand, the existence of " $U_{\text {ord }}$ " (respectively, " $U_{\mathrm{WP}}$ ") as above follows immediately from Lemma 6.3, (i) (respectively, Lemma 6.3, (ii)), together with a straightforward calculation. This completes the proof of Corollary 6.4.

Remark 6.4.1. Let us observe that the number "19" in the statement of Corollary 6.4 coincides with the result of the formula of [7], Chapter V, Corollary 1.3 , (3), pp. 237-238, i.e., the formula

$$
n_{2,0}^{\text {ord }}=\frac{p}{3}\left(2 p^{2}+1\right)
$$

Finally, we prove the existence of a nilpotent ordinary indigenous bundle over $X / S$ :

Proposition 6.5. The following hold:

(i) There exist at least 12 isomorphism classes of nilpotent ordinary indigenous bundles whose Hasse defects are nontrivial over $X / S$.

(ii) There exist at least 13 isomorphism classes of nilpotent ordinary indigenous bundles over $X / S$.

Proof. Let us identify $Q$ with $\mathbf{P}_{k}^{1}$ by an isomorphism over $k$. Moreover, let us naturally identify the set of closed points of $\mathbf{P}_{k}^{1}=Q$ with the set $k \cup\{\infty\}$.

First, I claim that the following assertion holds:

Claim 6.5.A. If the number " $n_{\mathrm{WP}}$ " defined in the statement of Corollary 6.2 is $\leq 12$, then, by considering a suitable automorphism of $\mathbf{P}_{k}^{1}=Q$, one may take the subset "WP" of $\mathbf{P}_{k}^{1}=Q$ to be

$$
\{0,1, \infty,-1, \alpha,-\alpha\} \subseteq k \cup\{\infty\}
$$


-where $\alpha \in k$ satisfies that $\alpha^{2}=2$-i.e., $X$ admits a dense open subscheme which is isomorphic to the affine scheme over $k$

$$
\operatorname{Spec}\left(k[s, t] /\left(s^{2}-f(t)\right)\right)
$$

- where $s$ and $t$ are indeterminates, and

$$
f(t)=x(x-1)(x+1)(x-\alpha)(x+\alpha)=x^{5}+2 x \in k[t] .
$$

Indeed, suppose that there exist 3 distinct subsets $D_{1}, D_{2}, D_{3} \subseteq \mathrm{WP}$ of cardinality 2 such that the elliptic curves $E_{D_{1}}, E_{D_{2}}$, and $E_{D_{3}}$ are not ordinary.

First, we consider the case where WP $=D_{1} \cup D_{2} \cup D_{3}$. Then let us observe that we may assume without loss of generality, by considering a suitable automorphism of $\mathbf{P}_{k}^{1}=Q$, that $\xi\left(D_{1}\right)=\{0, \infty\}$ and $1 \in \xi\left(D_{2}\right)$. Then since $E_{D_{2}}$ and $E_{D_{3}}$ are not ordinary, it follows from Lemma 6.3, (ii), that there exists an element $\alpha \in k \backslash\{0,1,-1\}$ such that

$$
\xi\left(D_{2}\right)=\{1,-1\}, \quad \xi\left(D_{3}\right)=\{\alpha,-\alpha\} .
$$

On the other hand, since

$$
\alpha \cdot(-\alpha)+\alpha \cdot 1+\alpha \cdot(-1)+(-\alpha) \cdot 1+(-\alpha) \cdot(-1)+1 \cdot(-1)=-\alpha^{2}-1,
$$

and $E_{D_{1}}$ is not ordinary, it follows from Lemma 6.3, (ii), that $\alpha^{2}=2$. Thus, one may take the subset "WP" to be as in Claim 6.5.A.

Next, we consider the case where WP $\neq D_{1} \cup D_{2} \cup D_{3}$. Then let us observe that we may assume without loss of generality, by considering a suitable automorphism of $\mathbf{P}_{k}^{1}=Q$, that $\infty \in \xi\left(\mathrm{WP} \backslash\left(D_{1} \cup D_{2} \cup D_{3}\right)\right)$, that $0 \in \xi\left(\mathrm{WP} \backslash\left(D_{1} \cup D_{2}\right)\right)$, and that $1 \in \xi\left(D_{1} \backslash\left(D_{1} \cap D_{2}\right)\right)$. Then since $E_{D_{1}}$ and $E_{D_{2}}$ are not ordinary, it follows from Lemma 6.3, (ii), that there exists an element $\alpha \in k \backslash\{0,1,-1\}$ such that

$$
\xi\left(\mathrm{WP} \backslash D_{1}\right)=\{0, \infty, \alpha,-\alpha\}, \quad \xi\left(\mathrm{WP} \backslash D_{2}\right)=\{0, \infty, 1,-1\},
$$

which thus implies that

$$
\xi(\mathrm{WP})=\{0,1,-1, \alpha,-\alpha, \infty\} .
$$

Thus, since $\infty \notin \xi\left(D_{3}\right)$, one verifies easily from Lemma 6.3 , (ii), that $E_{D_{3}}$ is ordinary-in contradiction to our assumption that $E_{D_{3}}$ is not ordinary. This completes the proof of Claim 6.5.A.

Now we verify assertion (i). Suppose that the number " $n_{\mathrm{WP}}$ " defined in the statement of Corollary 6.2 is $\leq 12$. Then it follows from Claim 6.5.A that one may take the subset "WP" to be as in Claim 6.5.A. In particular, it follows from Lemma 6.3, (ii), together with a straightforward calculation, that the number " $n_{\mathrm{WP}}$ " defined in the statement of Corollary 6.2 is equal to 12 . Thus, assertion (i) follows from Theorem 6.1. This completes the proof of assertion (i).

Next, we verify assertion (ii). Assume that the set of isomorphism classes of nilpotent ordinary indigenous bundles over $X / S$ is of cardinality $\leq 12$. Then it follows from Corollary 6.2 , (iii), that the number " $n_{\mathrm{WP}}$ " defined in the statement 
of Corollary 6.2 is $\leq 12$. Thus, it follows from Claim 6.5.A that one may take the subset "WP" to be as in Claim 6.5.A. Then it follows from Lemma 6.3, (ii), together with a straightforward calculation, that the number " $n_{\mathrm{WP}}$ " defined in the statement of Corollary 6.2 is equal to 12. Moreover, since [it follows from Lemma 6.3, (i), that] $X$ is parabolically ordinary, it follows from Corollary 6.2, (iii), that $X / S$ admits exactly $16(=12+4)>12$ isomorphism classes of nilpotent ordinary indigenous bundles - in contradiction to our assumption that the set of isomorphism classes of nilpotent ordinary indigenous bundles over $X / S$ is of cardinality $\leq 12$. This completes the proof of assertion (ii).

It follows from Proposition 6.5, (ii), together with [6], Chapter II, Proposition 3.4 , p. 1044, that the following corollary holds:

COROLlaRY 6.6. Every projective smooth curve of genus 2 over a connected noetherian scheme of characteristic 3 is hyperbolically ordinary [cf. Introduction].

Remark 6.6.1. Corollary 6.6 yields a partial positive answer to the basic question (1) of [7], Introduction, \$2.1, p. 72. By Corollary 6.6, we conclude that the image of the natural (1-)morphism discussed in the basic question (1) of [7], Introduction, $\$ 2.1$, p. 72 , in the case where $(g, r, p)=(2,0,3)$ contains the open substack $\left(\mathscr{M}_{2,0}\right)_{\mathbf{F}_{3}} \subseteq\left(\overline{\mathscr{M}}_{2,0}\right)_{\mathbf{F}_{3}}$.

Remark 6.6.2. Corollary 6.6 also yields an example of a projective smooth curve of positive characteristic which is not parabolically ordinary but hyperbolically ordinary [cf. also Lemma 6.3, (i)].

\section{Appendix A. Cartier operator associated to a square-trivialized invertible sheaf}

In the Appendix A, let us recall the Cartier operator associated to a squaretrivialized invertible sheaf on a projective smooth curve in positive characteristic. It seems to the author that the content of the Appendix A is well-known; however, since a suitable literature could not be found, the author has decided to discuss it in the Appendix A.

In the Appendix A, let $p$ be an odd prime number, $g \geq 2$ an integer, $S$ a connected noetherian scheme of characteristic $p$ [i.e., over $\mathbf{F}_{p}$ ], and $f: X \rightarrow S$ a projective smooth curve [i.e., a morphism which is projective, smooth, geometrically connected, and of relative dimension one] of genus $g$. Write $f^{F}: X^{F} \rightarrow S$ for the projective smooth curve obtained by base-changing $f$ via the absolute Frobenius morphism of $S$ and $\Phi: X \rightarrow X^{F}$ for the relative Frobenius morphism over $S$. We shall use the notation " $\omega$ " (respectively, " $\tau$ ") to denote the relative cotangent (respectively, tangent) sheaf.

Proposition A.1. Let $\mathscr{L}$ be an invertible sheaf on $X$ such that $\mathscr{L}^{\otimes 2} \cong \mathcal{O}_{X}$. Then the following conditions are equivalent: 
(1) The restriction of $\mathscr{L}$ to every fiber of $f$ is of order two [i.e., is nontrivial].

(2) There exists a point $s \in S$ of $S$ such that the restriction of $\mathscr{L}$ to the fiber of $f$ at $s$ is of order two [i.e., is nontrivial].

(3) The invertible sheaf $\mathscr{L}$ does not arise from an invertible sheaf on $S$.

(4) The image of the classifying morphism of $\mathscr{L}[$ from $S$ to the relative Jacobian variety of $X / S]$ does not intersect the image of the identity section.

Proof. The implications $(1) \Rightarrow(2) \Rightarrow(3)$ and $(4) \Rightarrow(1)$ are immediate. The implication $(3) \Rightarrow(4)$ follows immediately from our assumption that $S$ is connected, together with the [well-known] fact that the endomorphism of the relative Jacobian variety of $X / S$ obtained by multiplication by 2 is finite étale. This completes the proof of Proposition A.1.

Definition A.2. Let $\mathscr{L}$ be an invertible sheaf on $X$ such that $\mathscr{L}^{\otimes 2} \cong \mathcal{O}_{X}$. Then we shall say that $\mathscr{L}$ is of relative order two (respectively, one) if $\mathscr{L}$ satisfies (respectively, does not satisfy) the four conditions in the statement of Proposition A.1.

Definition A.3. We shall refer to a pair

$$
\left(\mathscr{L}, \Theta: \mathscr{L}^{\otimes 2} \stackrel{\sim}{\rightarrow} \mathcal{O}_{X}\right)
$$

consisting of an invertible sheaf $\mathscr{L}$ on $X$ and a global trivialization $\Theta$ of the square $\mathscr{L}^{\otimes 2}$ of $\mathscr{L}$ as a square-trivialized invertible sheaf on $X$.

Let

$$
\mathscr{L}=\left(\mathscr{L}, \Theta: \mathscr{L}^{\otimes 2} \stackrel{\sim}{\rightarrow} \mathcal{O}_{X}\right)
$$

be a square-trivialized invertible sheaf on $X$. Thus, the trivialization $\Theta$ determines isomorphisms of invertible sheaves on $X$

$$
\begin{aligned}
\mathscr{L} & \stackrel{\sim}{\rightarrow} \mathscr{L}^{\otimes p} \stackrel{\sim}{\rightarrow} \Phi^{*} \mathscr{L}^{F} \\
\Theta(l \otimes l)^{(p-1) / 2} \cdot l & \mapsto l^{\otimes p} \mapsto \Phi^{-1} l^{F}
\end{aligned}
$$

- where we write $\mathscr{L}^{F}$ for the invertible sheaf on $X^{F}$ obtained by pulling back $\mathscr{L}$ via the morphism $X^{F} \rightarrow X$ induced by the absolute Frobenius morphism of $S, l$ is a local section of $\mathscr{L}$, and $l^{F}$ is the local section of $\mathscr{L}^{F}$ determined by $l$.

Let us recall [cf., e.g., [4], Theorem 7.2] that we have an exact sequence of $\mathcal{O}_{X^{F}}$-modules

$$
0 \longrightarrow \mathcal{O}_{X^{F}} \longrightarrow \Phi_{*} \mathcal{O}_{X} \stackrel{\Phi_{*} d}{\longrightarrow} \Phi_{*} \omega_{X / S} \stackrel{c}{\longrightarrow} \omega_{X^{F} / S} \longrightarrow 0
$$

- where we write $d$ for the exterior differentiation operator $\mathcal{O}_{X} \rightarrow \omega_{X / S}$, and $c$ for the Cartier operator. We shall write

$$
\mathscr{B}_{\circ} \stackrel{\text { def }}{=} \operatorname{Coker}\left(\mathcal{O}_{X^{F}} \rightarrow \Phi_{*} \mathcal{O}_{X}\right)=\operatorname{Ker}\left(c: \Phi_{*} \omega_{X / S} \rightarrow \omega_{X^{F} / S}\right)
$$


for the locally free coherent $\mathcal{O}_{X^{F}}$-module of rank $p-1$ obtained by forming the cokernel of the natural homomorphism $\mathcal{O}_{X}{ }^{F} \rightarrow \Phi_{*} \mathcal{O}_{X}$, or, alternatively, the kernel of the Cartier operator $c: \Phi_{*} \omega_{X / S} \rightarrow \omega_{X^{F} / S}$. Then, by tensoring with $\mathscr{L}^{F}$ and applying the above isomorphism $\mathscr{L} \stackrel{\sim}{\rightarrow} \Phi^{*} \mathscr{L}^{F}$ determined by $\Theta$, we obtain an exact sequence of $\mathcal{O}_{X^{F}}$-modules

$$
0 \rightarrow \mathscr{L}^{F} \otimes_{\mathcal{O}_{X} F} \mathscr{B}_{\circ} \rightarrow \Phi_{*}\left(\mathscr{L} \otimes_{\mathcal{O}_{X}} \omega_{X / S}\right) \rightarrow \mathscr{L}^{F} \otimes_{\mathcal{O}_{X} F} \omega_{X^{F} / S} \rightarrow 0,
$$

which thus determines an exact sequence of $\mathcal{O}_{S}$-modules

$$
0 \rightarrow f_{*}^{F}\left(\mathscr{L}^{F} \otimes_{\mathcal{O}_{X} F} \mathscr{B}_{\circ}\right) \rightarrow f_{*}\left(\mathscr{L} \otimes_{\mathcal{O}_{X}} \omega_{X / S}\right) \rightarrow f_{*}^{F}\left(\mathscr{L}^{F} \otimes_{\mathcal{O}_{X} F} \omega_{X^{F} / S}\right) .
$$

Definition A.4. We shall write

$$
C_{\mathscr{L}}
$$

for the third arrow of the above exact sequence of $\mathcal{O}_{S}$-modules and refer to

$$
C_{\mathscr{L}}: f_{*}\left(\mathscr{L} \otimes_{\mathcal{O}_{X}} \omega_{X / S}\right) \rightarrow f_{*}^{F}\left(\mathscr{L}^{F} \otimes_{\mathcal{O}_{X} F} \omega_{X^{F} / S}\right)
$$

as the Cartier operator associated to $\mathscr{L}=(\mathscr{L}, \Theta)$.

Remark A.4.1. If we take the pair " $(\mathscr{L}, \Theta)$ " to be the pair consisting of $\mathcal{O}_{X}$ and the natural identification $\mathcal{O}_{X} \otimes_{\mathcal{O}_{X}} \mathcal{O}_{X}=\mathcal{O}_{X}$, then the Cartier operator $f_{*} \omega_{X / S}$ $\rightarrow f_{*}^{F} \omega_{X^{F} / S}$ associated to $(\mathscr{L}, \Theta)$ coincides with the [homomorphism induced by the] usual Cartier operator.

Remark A.4.2. One verifies easily that since the formation of

$$
0 \longrightarrow \mathcal{O}_{X}{ }^{F} \longrightarrow \Phi_{*} \mathcal{O}_{X} \stackrel{\Phi_{*} d}{\longrightarrow} \Phi_{*} \omega_{X / S} \stackrel{c}{\longrightarrow} \omega_{X^{F} / S} \longrightarrow 0
$$

commutes with arbitrary change of base " $S^{\prime} \rightarrow S$ ", the formation of

$$
C_{\mathscr{L}}: f_{*}\left(\mathscr{L} \otimes_{\mathcal{O}_{X}} \omega_{X / S}\right) \rightarrow f_{*}^{F}\left(\mathscr{L}^{F} \otimes_{\mathcal{O}_{X} F} \omega_{X^{F} / S}\right)
$$

commutes with arbitrary change of base " $S$ ' $\rightarrow$ ". write

If [the underlying invertible sheaf of] $\mathscr{L}$ is of relative order two, then we shall

$$
\xi_{\mathscr{L}}: X_{\mathscr{L}} \rightarrow X
$$

for the connected finite étale double covering of $X$ over $S$ which trivializes the invertible sheaf $\mathscr{L}$ [determined by $\Theta$ ] and

$$
\xi_{\mathscr{L}}^{F}: X_{\mathscr{L}}^{F} \rightarrow X^{F}
$$

for the connected finite étale covering of $X^{F}$ over $S$ obtained by base-changing $\xi_{\mathscr{L}}$ via the absolute Frobenius morphism of $S$. Thus, a trivialization of $\xi_{\mathscr{L}}^{*} \mathscr{L}$ determines respective isomorphisms of $\mathcal{O}_{X^{-}}, \mathcal{O}_{X^{F}}$-modules

$$
\left(\xi_{\mathscr{L}}\right)_{*} \mathcal{O}_{X_{\mathscr{L}}} \cong \mathcal{O}_{X} \oplus \mathscr{L}, \quad\left(\xi_{\mathscr{L}}^{F}\right)_{*} \mathcal{O}_{X_{\mathscr{L}}^{F}} \cong \mathcal{O}_{X^{F}} \oplus \mathscr{L}^{F}
$$


NILPOTENT ADMISSIBLE INDIGENOUS BUNDLES IN CHARACTERISTIC THREE 723

Moreover, one verifies immediately that the natural homomorphism of $\mathcal{O}_{S^{-}}$ modules

$$
\mathbf{R}^{1}\left(f^{F} \circ \xi_{\mathscr{L}}^{F}\right)_{*} \mathcal{O}_{X_{\mathscr{L}}^{F}} \rightarrow \mathbf{R}^{1}\left(f \circ \xi_{\mathscr{L}}\right)_{*} \mathcal{O}_{X_{\mathscr{L}}}
$$

determined by the relative Frobenius morphism $X_{\mathscr{L}} \rightarrow X_{\mathscr{L}}^{F}$ over $S$ is decomposed into the direct sum of the natural homomorphisms of $\mathcal{O}_{S}$-modules

$$
\mathbf{R}^{1} f_{*}^{F} \mathcal{O}_{X^{F}} \rightarrow \mathbf{R}^{1} f_{*} \mathcal{O}_{X}, \quad \mathbf{R}^{1} f_{*}^{F} \mathscr{L}^{F} \rightarrow \mathbf{R}^{1} f_{*} \Phi^{*} \mathscr{L}^{F} \stackrel{\sim}{\leftarrow} \mathbf{R}^{1} f_{*} \mathscr{L}
$$

[cf. the isomorphism given in the discussion following Definition A.3].

DEFINITION A.5.

(i) We shall say that $f: X \rightarrow S$ is parabolically ordinary [cf. the discussion following [6], Chapter II, Definition 3.3, p. 1044] if the Jacobian variety of every fiber of $f$ is ordinary.

(ii) Let $Y \rightarrow S$ be a projective smooth curve over $S$ and $\xi: Y \rightarrow X$ a finite étale covering over $S$. Then we shall say that $\xi: Y \rightarrow X$ is parabolically newordinary if, for every point $s \in S$ of $S$, the "new part" of $\xi$ at $s$ [i.e., the abelian variety over $s$ obtained by forming the quotient of the Jacobian variety of $Y \times_{S} s$ by the image - via the homomorphism induced by $\xi$ - of the Jacobian variety of $X \times s s]$ is ordinary.

Thus, we obtain:

Proposition A.6. It holds that the Cartier operator $C_{\mathscr{L}}: f_{*}\left(\mathscr{L} \otimes_{\mathcal{O}_{X}} \omega_{X / S}\right) \rightarrow$ $f_{*}^{F}\left(\mathscr{L}^{F} \otimes_{\mathcal{O}_{X} F} \omega_{X^{F} / S}\right)$ associated to $\mathscr{L}=(\mathscr{L}, \Theta)$ is injective at every point of $S$ if and only if one of the following conditions is satisfied:

(1) $\mathscr{L}$ is of relative order one, and $X / S$ is parabolically ordinary.

(2) $\mathscr{L}$ is of relative order two, and the connected finite étale double covering $\xi_{\mathscr{L}}: X_{\mathscr{L}} \rightarrow X$ is parabolically new-ordinary.

Proof. Let us first observe that it follows from Remark A.4.2 that, to verify Proposition A.6, we may assume without loss of generality that $S$ is the spectrum of an algebraically closed field. Next, let us recall from the discussion preceding Definition A.4 that we have an isomorphism of $\mathcal{O}_{S}$-modules

$$
f_{*}^{F}\left(\mathscr{L}^{F} \otimes_{\mathcal{O}_{X} F} \mathscr{B}_{\circ}\right) \stackrel{\sim}{\rightarrow} \operatorname{Ker}\left(C_{\mathscr{L}}\right) .
$$

In particular, the exact sequence of $\mathcal{O}_{X^{F}}$-modules in the discussion preceding Definition A.4

$$
0 \longrightarrow \mathcal{O}_{X^{F}} \longrightarrow \Phi_{*} \mathcal{O}_{X} \stackrel{\Phi_{*} d}{\longrightarrow} \Phi_{*} \omega_{X / S} \stackrel{c}{\longrightarrow} \omega_{X^{F} / S} \longrightarrow 0,
$$

together with the isomorphism $\mathscr{L} \stackrel{\sim}{\rightarrow} \Phi^{*} \mathscr{L}^{F}$ given in the discussion following Definition A.3, determines an exact sequence of $\mathcal{O}_{S}$-modules

$$
0 \rightarrow \operatorname{Ker}\left(C_{\mathscr{L}}\right) \rightarrow \mathbf{R}^{1} f_{*}^{F} \mathscr{L}^{F} \rightarrow \mathbf{R}^{1} f_{*} \mathscr{L} .
$$


Thus, it follows from the discussion preceding Definition A.5 that Proposition A.6 holds. This completes the proof of Proposition A.6.

Definition A.7. Let $\mathscr{M}$ be an invertible sheaf on $X$ such that $\mathscr{M}^{\otimes 2} \cong \mathcal{O}_{X}$. Then we shall say that $\mathscr{M}$ is parabolically ordinary if, for some [or, equivalently, every] trivialization $\Theta_{\mathscr{M}}: \mathscr{M}^{\otimes 2} \stackrel{\sim}{\rightarrow} \mathcal{O}_{X}$, the square-trivialized invertible sheaf $\left(\mathscr{M}, \Theta_{\mathscr{M}}\right)$ satisfies either (1) or (2) in the statement of Proposition A.6 [i.e., the Cartier operator associated to $\left(\mathscr{M}, \Theta_{\mathscr{M}}\right)$ is injective at every point of $S$-cf. Proposition A.6].

Next, let us observe that the morphism $X^{F} \rightarrow X$ induced by the absolute Frobenius morphism of $S$ determines a Frobenius-semi-linear homomorphism

$$
f_{*}\left(\mathscr{L} \otimes_{\mathcal{O}_{X}} \omega_{X / S}\right) \rightarrow f_{*}^{F}\left(\mathscr{L}^{F} \otimes_{\mathcal{O}_{X} F} \omega_{X^{F} / S}\right) .
$$

For a global section $u$ of $\mathscr{L} \otimes_{\mathcal{O}_{X}} \omega_{X / S}$, we shall write $u^{F}$ for the global section of $\mathscr{L}^{F} \otimes_{\mathcal{O}_{X F}} \omega_{X^{F} / S}$ obtained by forming the image of $u$ via this Frobenius-semilinear homomorphism.

DeFINITION A.8.

(i) We shall say that a global section $u$ of $\mathscr{L} \otimes_{\mathcal{O}_{X}} \omega_{X / S}$ is a normalized Cartier eigenform associated to $\mathscr{L}=(\mathscr{L}, \Theta)$ if $u$ defines a relative effective Cartier divisor of $X / S$, and, moreover, $C_{\mathscr{L}}(u)=-u^{F}$.

(ii) Let $\mathscr{M}$ be an invertible sheaf on $X$ such that $\mathscr{M}^{\otimes 2} \cong \mathcal{O}_{X}$. Then we shall say that a global section of $\mathscr{M} \otimes_{\mathcal{O}_{X}} \omega_{X / S}$ is a Cartier eigenform associated to $\mathscr{M}$ if there exists a trivialization $\Theta_{\mathscr{M}}$ of the square of $\mathscr{M}$ such that the global section is a normalized Cartier eigenform associated to the square-trivialized invertible sheaf $\left(\mathscr{M}, \Theta_{\mathscr{M}}\right)$.

Remark A.8.1. One verifies immediately that if $S$ is the spectrum of an algebraically closed field $k$ [of characteristic $p$ ], then the following two conditions are equivalent:

- A global section $u \in \Gamma\left(X, \mathscr{L} \otimes_{\mathcal{O}_{X}} \omega_{X / S}\right)$ is a Cartier eigenform associated to [the underlying invertible sheaf of] $\mathscr{L}$.

- It holds that $u \neq 0$, and, moreover, $C_{\mathscr{L}}(u)$ is a $k^{\times}$-multiple of $u^{F}$. Moreover, in this case, the subset of $k^{\times}$consisting of $c \in k^{\times}$such that $c u$ is a normalized Cartier eigenform associated to $\mathscr{L}=(\mathscr{L}, \Theta)$ forms an $\mathbf{F}_{p}^{\times}$-torsor, which thus implies that this subset is of cardinality $p-1$.

Remark A.8.2. If we take the pair " $(\mathscr{L}, \Theta)$ " to be the pair consisting of $\mathcal{O}_{X}$ and the natural identification $\mathcal{O}_{X} \otimes_{\mathcal{O}_{X}} \mathcal{O}_{X}=\mathcal{O}_{X}$ [i.e., if we are in the situation of Remark A.4.1], then the property of being a [normalized] Cartier eigenform is closely related to the property of being locally logarithmic [cf., e.g., [3], Théorème 2.1.17]. 
Finally, we consider a local criterion for a normalized Cartier eigenform. Let $x \in X$ be a point of $X, t_{x}=t \in \mathcal{O}_{X}$ a local parameter of $X / S$ at $x, l_{x}=l \in \mathscr{L}$ a local trivialization of $\mathscr{L}$ at $x$, and

$$
\chi \in \Gamma\left(X, \mathscr{L} \otimes_{\mathcal{O}_{X}} \omega_{X / S}\right)
$$

a global section of $\mathscr{L} \otimes_{\mathcal{O}_{X}} \omega_{X / S}$. Then the global trivialization $\Theta$ and the local trivialization $l_{x}=l$ determine a local unit

$$
\delta_{x}=\delta \stackrel{\text { def }}{=} \Theta(l \otimes l) \in \mathcal{O}_{X}^{\times}
$$

at $x$. Moreover, the global section $\chi$ determines a local function $\phi_{x}=\phi \in \mathcal{O}_{X}$ on $X$ at $x$ which fits into the equality

$$
\chi=\phi \cdot l \otimes d t
$$

at $x$. Then it follows immediately from the characterization of the Cartier operator [cf., e.g., [4], Theorem 7.2; also the discussion given in [3], §2.1especially, the equality $(2.1 .13)$ in [3], \$2.1], together with the explicit description of the isomorphism $\mathscr{L} \stackrel{\sim}{\rightarrow} \Phi^{*} \mathscr{L}^{F}$ given in the discussion following Definition A.3, that the following lemma holds:

Lemma A.9. Write $\partial_{t_{x}}$ for the local derivation corresponding to the local trivialization of $\tau_{X / S}$ which maps $d t_{x}$ to 1 [i.e., " $\partial_{t_{x}}(-)$ " is the "derivative of $(-)$ with respect to $t_{x}$ "]. Then the following hold:

(i) It holds that the global section $\chi$ of $\mathscr{L} \otimes_{\mathcal{O}_{X}} \omega_{X / S}$ is annihilated by the Cartier operator $C_{\mathscr{L}}$ associated to $\mathscr{L}=(\mathscr{L}, \Theta)$ if and only if, for every point $x \in X$, the equality

$$
(\overbrace{\partial_{t_{x}} \circ \cdots \circ \partial_{t_{x}}}^{p-1})\left(\delta_{x}^{-(p-1) / 2} \cdot \phi_{x}\right)=0
$$

holds.

(ii) It holds that the global section $\chi$ of $\mathscr{L} \otimes_{\mathcal{O}_{X}} \omega_{X / S}$ is a normalized Cartier eigenform associated to $\mathscr{L}=(\mathscr{L}, \Theta)$ if and only if, for every point $x \in X$, the local function $\phi_{x}$ is not a zero-divisor, and, moreover, the equality

$$
\phi_{x}^{p}=(\overbrace{\partial_{t_{x}} \circ \cdots \circ \partial_{t_{x}}}^{p-1})\left(\delta_{x}^{-(p-1) / 2} \cdot \phi_{x}\right)
$$

holds.

\section{Appendix B. The Hasse bundle of a nilpotent admissible indigenous bundle}

In the Appendix B, we discuss the Hasse bundle of a nilpotent admissible indigenous bundle. In the Appendix $\mathrm{B}$, let $p$ be an odd prime number, $g \geq 2$ an integer, $S$ a connected noetherian scheme of characteristic $p$ [i.e., over $\mathbf{F}_{p}$ ], and $f: X \rightarrow S$ a projective smooth curve [i.e., a morphism which is projective, 
smooth, geometrically connected, and of relative dimension one] of genus $g$. Write $f^{F}: X^{F} \rightarrow S$ for the projective smooth curve obtained by base-changing $f$ via the absolute Frobenius morphism of $S$ and $\Phi: X \rightarrow X^{F}$ for the relative Frobenius morphism over $S$. We shall use the notation " $\omega$ " (respectively, " $\tau$ ") to denote the relative cotangent (respectively, tangent) sheaf.

Let

$$
P=\left(\pi: P \rightarrow X, \nabla_{P}\right)
$$

be a nilpotent admissible indigenous bundle over $X / S$. Write

$$
\sigma_{\mathrm{Hdg}}: X \rightarrow P
$$

for the Hodge section of $P$ and

$$
\mathscr{I}_{\mathrm{Hdg}} \subseteq \mathcal{O}_{P}
$$

for the ideal of $\mathcal{O}_{P}$ which defines the section $\sigma_{\mathrm{Hdg}}$. Thus, it follows from the definition of an indigenous bundle that the Kodaira-Spencer homomorphism at $\sigma_{\mathrm{Hdg}}$ relative to $\nabla_{P}$ [i.e., the homomorphism obtained by differentiating $\sigma_{\mathrm{Hdg}}$ by means of $\nabla_{P}$ ]

$$
\sigma_{\mathrm{Hdg}}^{*} \omega_{P / X} \rightarrow \omega_{X / S}
$$

is an isomorphism.

Proposition B.1. There exists a unique section $\sigma_{\mathrm{con}}: X \rightarrow P$ of $\pi: P \rightarrow X$ which satisfies the following conditions:

(1) The section $\sigma_{\mathrm{con}}$ is horizontal with respect to $\nabla_{P}$. In particular, the connection $\nabla_{P}$ induces a connection on the invertible sheaf $\sigma_{\text {con }}^{*} \omega_{P / X}$ on $X$.

(2) There exists a horizontal isomorphism $\sigma_{\mathrm{con}}^{*} \omega_{P / X} \cong \Phi^{*} \tau_{X^{F} / S}$, where we regard $\Phi^{*} \tau_{X^{F} / S}$ as an invertible sheaf equipped with a connection by equipping $\Phi^{*} \tau_{X^{F} / S}$ with the connection arising from the exterior differentiation operator $\mathcal{O}_{X} \rightarrow \omega_{X / S}$.

We shall refer to this section $\sigma_{\mathrm{con}}$ as the conjugate section of the indigenous bundle $P$.

Proof. This follows from the second paragraph of [the statement of] [6], Chapter II, Proposition 2.5, pp. 1030-1031.

Write

$$
\sigma_{\mathrm{con}}: X \rightarrow P
$$

for the conjugate section of $P$ and

$$
\mathscr{I}_{\text {con }} \subseteq \mathcal{O}_{P}
$$

for the ideal of $\mathcal{O}_{P}$ which defines the section $\sigma_{\text {con }}$. 
Definition B.2. We shall refer to the invertible sheaf on $X$

$$
\sigma_{\mathrm{Hdg}}^{*} \mathscr{H}_{0 m_{\mathcal{O}_{P}}}\left(\mathscr{I}_{\text {con }}, \mathcal{O}_{P}\right)=\mathscr{H}_{0 m_{\mathcal{O}_{X}}}\left(\sigma_{\text {Hdg }}^{*} \mathscr{I}_{\text {con }}, \mathcal{O}_{X}\right)
$$

obtained by pulling back $\mathscr{H}_{0 m_{\mathcal{O}_{P}}}\left(\mathscr{I}_{\text {con }}, \mathcal{O}_{P}\right)$ via $\sigma_{\mathrm{Hdg}}$ as the Hasse bundle of $P$. We shall refer to the invertible sheaf on $X$

$$
\sigma_{\mathrm{Hdg}}^{*} \mathscr{H}_{o m_{\mathcal{O}_{P}}}\left(\mathscr{I}_{\text {con }}, \mathcal{O}_{P}\right) \otimes_{\mathcal{O}_{X}} \tau_{X / S}^{\otimes(p-1) / 2}=\mathscr{H}^{\otimes} m_{\mathcal{O}_{X}}\left(\sigma_{\mathrm{Hdg}}^{*} \mathscr{I}_{\text {con }}, \tau_{X / S}^{\otimes(p-1) / 2}\right)
$$

obtained by tensoring the Hasse bundle with $\tau_{X / S}^{\otimes(p-1) / 2}$ as the Hasse defect of $P$.

Write

$$
\mathscr{H}_{P} \stackrel{\text { def }}{=} \sigma_{\mathrm{Hdg}}^{*} \mathscr{H}_{0} m_{\mathcal{O}_{P}}\left(\mathscr{I}_{\text {con }}, \mathcal{O}_{P}\right)
$$

for the Hasse bundle of $P$. Then let us observe that since $\pi: P \rightarrow X$ is of genus zero, and the invertible sheaf on $P$

$$
\mathscr{H}_{\text {om }}\left(\mathscr{I}_{\mathrm{con}}, \mathscr{I}_{\mathrm{Hdg}}\right)
$$

is of relative degree 0 over $X$, it follows immediately that the natural homomorphisms

$$
\begin{aligned}
& \pi_{*} \mathscr{H}_{0 \mathrm{O}_{\mathcal{O}_{P}}}\left(\mathscr{I}_{\mathrm{con}}, \mathscr{I}_{\mathrm{Hdg}}\right) \rightarrow \sigma_{\mathrm{Hdg}}^{*} \mathscr{H}_{o m_{\mathcal{P}_{P}}}\left(\mathscr{I}_{\text {con }}, \mathscr{I}_{\mathrm{Hdg}}\right) \cong \mathscr{H}_{P} \otimes_{\mathcal{O}_{X}} \omega_{X / S},
\end{aligned}
$$

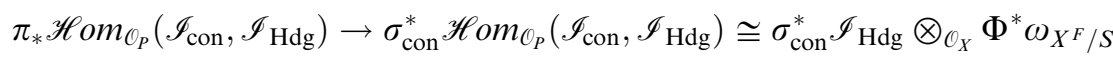

[cf. the discussion preceding Proposition B.1; Proposition B.1, (2)] are isomorphisms. Thus, by means of the natural identification $\sigma_{\mathrm{Hdg}}^{*} \mathscr{I}_{\text {con }}=\sigma_{\text {con }}^{*} \mathscr{I}_{\mathrm{Hdg}}$, we obtain:

Proposition B.3. There exist isomorphisms of invertible sheaves on $X$

$$
\mathscr{H}_{P}^{\otimes 2} \cong \mathscr{H}_{0 m_{\mathcal{O}_{X}}}\left(\Phi^{*} \tau_{X^{F} / S}, \tau_{X / S}\right) \cong \omega_{X / S}^{\otimes p-1} .
$$

In particular, the square of the Hasse defect is trivial.

Moreover, we obtain:

Proposition B.4. The global section of

$$
\mathscr{H}_{o m_{\mathcal{O}_{X}}}\left(\Phi^{*} \tau_{X^{F} / S}, \tau_{X / S}\right)
$$

obtained, relative to the isomorphism of Proposition B.3, by forming the square of the global section of $\mathscr{H}_{P}$ determined by the natural inclusion $\mathscr{I}_{\text {con }} \hookrightarrow \mathcal{O}_{P}$ coincides, up to multiplication by a global section of $\mathcal{O}_{S}^{\times}$, with the square Hasse invariant of $P$. In particular, the global section of $\mathscr{H}_{P}$ determined by the natural inclusion $\mathscr{I}_{\text {con }} \hookrightarrow \mathcal{O}_{P}$ coincides, up to multiplication by a global section of $\mathcal{O}_{S}^{\times}$, with the Hasse invariant of $P$.

Proof. This follows from the discussion in the proof of [6], Chapter II, Proposition 2.6, (3), p. 1032. 


\section{Appendix C. Various moduli stacks}

In the Appendix $\mathrm{C}$, we consider various moduli stacks related to the notions discussed in the present paper. In the Appendix $\mathrm{C}$, let $p$ be an odd prime number and $g \geq 2$ an integer.

We shall write

$$
\mathscr{M}_{g}
$$

for the moduli stack of projective smooth curves of genus $g$ of characteristic $p$;

$$
\mathscr{C}_{g} \rightarrow \mathscr{M}_{g}
$$

for the universal curve over $\mathscr{M}_{g}$;

$$
\mathscr{J}_{g} \rightarrow \mathscr{M}_{g}
$$

for the relative Jacobian variety of $\mathscr{C}_{g} \rightarrow \mathscr{M}_{g}$;

$$
\mathscr{J}_{g}[n] \subseteq \mathscr{J}_{g}
$$

for the kernel of the endomorphism of $\mathscr{J}_{g}$ over $\mathscr{M}_{g}$ obtained by multiplication by $n$ [where $n$ is a nonnegative integer]. Moreover, we shall write

$$
\mathscr{N}_{g}
$$

for the moduli stack of smooth nilcurves of genus $g$ of characteristic $p$, i.e., the moduli stack of projective smooth curves of genus $g$ of characteristic $p$ equipped with nilpotent indigenous bundles;

$$
\mathscr{N}_{g}^{\mathrm{adm}} \subseteq \mathscr{N}_{g}
$$

for the moduli stack of projective smooth curves of genus $g$ of characteristic $p$ equipped with nilpotent admissible indigenous bundles;

$$
\mathscr{N}_{g}^{\text {ord }} \subseteq \mathscr{N}_{g}^{\text {adm }}
$$

for the moduli stack of projective smooth curves of genus $g$ of characteristic $p$ equipped with nilpotent ordinary indigenous bundles.

Definition C.1. It follows from the final portion of Proposition B.3 that the Hasse defect of the universal nilpotent admissible indigenous bundle over $\mathscr{C}_{g} \times \mathscr{M}_{g} \mathscr{N}_{g}^{\text {adm }} \rightarrow \mathscr{N}_{g}^{\text {adm }}$ determines a (1-)morphism over $\mathscr{M}_{g}$

$$
\mathscr{N}_{g}^{\mathrm{adm}} \rightarrow \mathscr{J}_{g}[2] \text {. }
$$

We shall refer to this (1-)morphism as the Hasse defect morphism.

Proposition C.2. The following three open substacks of $\mathscr{N}_{g}^{\mathrm{adm}}$ coincide:

(1) The open substack $\mathscr{N}_{g}^{\text {ord }} \subseteq \mathscr{N}_{g}^{\text {adm }}$.

(2) The étale locus of the natural (1-)morphism $\mathscr{N}_{g}^{\mathrm{adm}} \rightarrow \mathscr{M}_{g}$.

(3) The étale locus of the Hasse defect morphism $\mathscr{N}_{g}^{\mathrm{adm}} \rightarrow \mathscr{J}_{g}[2]$. 
Proof. The assertion that the open substack given in (1) coincides with the open substack given in (2) follows from the definition [cf. also the discussion following [7], Introduction, Theorem 0.1, p. 24]. On the other hand, since the (1-)morphism $\mathscr{N}_{g}^{\mathrm{adm}} \rightarrow \mathscr{M}_{g}$ is flat [cf. [6], Chapter II, Theorem 2.3, p. 1029], the assertion that the open substack given in (2) coincides with the open substack given in (3) follows from the well-known fact that the natural (1-)morphism $\mathscr{J}_{g}[2] \rightarrow \mathscr{M}_{g}$ is a finite étale surjection.

Now let us observe that since, as is well-known, $\mathscr{J}_{g}[2]$ is finite étale over $\mathscr{M}_{g}$, the identity section of $\mathscr{J}_{g} \rightarrow \mathscr{M}_{g}$ determines an isomorphism of stacks over $\mathscr{M}_{g}$

$$
\mathscr{M}_{g} \sqcup\left(\mathscr{J}_{g}[2] \backslash \mathscr{J}_{g}[1]\right) \stackrel{\sim}{\rightarrow} \mathscr{J}_{g}[2] .
$$

Thus, by considering the Hasse defect morphism, we obtain:

Proposition C.3. Let $U \rightarrow \mathscr{N}_{g}^{\mathrm{adm}}$ be a scheme over $\mathscr{N}_{g}^{\mathrm{adm}}$. Suppose that there exist two geometric points $s_{1}, s_{2}$ of $U$ such that the Hasse defect of the nilpotent admissible indigenous bundle corresponding to $s_{1}$ (respectively, $s_{2}$ ) is of relative order one (respectively, two). Then $U$ is not connected.

Next, we shall write

$$
\mathscr{R}_{g}
$$

for the moduli stack of "nontrivial" smooth Prym curves of genus $g$ of characteristic $p$, i.e., the moduli stack of projective smooth curves of genus $g$ of characteristic $p$ equipped with square-trivialized invertible sheaves whose underlying invertible sheaves are of relative order two;

$$
\underline{\mathscr{R}}_{g} \stackrel{\text { def }}{=} \mathscr{J}_{g}[2] \backslash \mathscr{F}_{g}[1] \text {. }
$$

Thus, we have a natural (1-)morphism $\mathscr{R}_{g} \rightarrow \underline{\mathscr{R}}_{g}$ over $\mathscr{M}_{g}$. For a nonnegative integer $d$, write

$$
\mathscr{A}_{d}
$$

for the moduli stack of principally polarized abelian varieties of dimension $d$ of characteristic $p$ and

$$
\mathscr{A}_{d}^{\text {ord }} \subseteq \mathscr{A}_{d}
$$

for the moduli stack of principally polarized ordinary abelian varieties of dimension $d$ of characteristic $p$.

Definition C.4. Since $\mathscr{M}_{g} \sqcup \mathscr{\mathscr { R }}_{g}$ is naturally isomorphic to $\mathscr{J}_{g}[2]$ over $\mathscr{M}_{g}$, the inverse image of $\mathscr{A}_{g}^{\text {ord }} \subseteq \mathscr{A}_{g}$ via the Torelli morphism $\mathscr{M}_{g} \rightarrow \mathscr{A}_{g}$ and the 
image in $\mathscr{R}_{g}$ of the inverse image of $\mathscr{A}_{g-1}^{\text {ord }} \subseteq \mathscr{A}_{g-1}$ via the Prym morphism $\mathscr{R}_{g} \rightarrow \mathscr{A}_{g-1}$ determine an open substack of $\mathscr{J}_{g}[2]$. We shall write

$$
\mathscr{J}_{g}[2]^{\text {pb-ord }} \subseteq \mathscr{J}_{g}[2]
$$

for this open substack.

Thus, it follows immediately from the various definitions involved that the following proposition holds:

Proposition C.5. In the notation introduced at the beginning of the Appendix $\mathrm{B}$, let $P$ be a nilpotent admissible indigenous bundle over $X / S$. Then the following conditions are equivalent:

(1) The image of the composite

$$
S \rightarrow \mathscr{N}_{g}^{\mathrm{adm}} \rightarrow \mathscr{J}_{g}[2]
$$

of the classifying (1-)morphism $S \rightarrow \mathscr{N}_{g}^{\mathrm{adm}}$ of $P$ and the Hasse defect morphism is contained in the open substack $\mathscr{J}_{g}[2]^{\text {pb-ord }} \subseteq \mathscr{J}_{g}[2]$.

(2) The Hasse defect of $P$ is parabolically ordinary.

Acknowledgments. The author would like to thank Shinichi Mochizuki, Yasuhiro Wakabayashi, and the referee for comments concerning the proof of Theorem 2.1 and the content of Remark 2.1.1. This research was supported by Grant-in-Aid for Scientific Research (C), No. 24540016, Japan Society for the Promotion of Science.

\section{REFERENCES}

[1] L. R. A. Finotti, Minimal degree liftings of hyperelliptic curves, J. Math. Sci. Univ. Tokyo 11 (2004), 1-47.

[2] R. C. Gunning, Special coordinate coverings of Riemann surfaces, Math. Ann. 170 (1967), 67-86.

[ 3 ] L. Illusie, Complexe de deRham-Witt et cohomologie cristalline, Ann. Sci. École Norm. Sup. (4) 12 (1979), 501-661.

[4] N. M. Katz, Nilpotent connections and the monodromy theorem: Applications of a result of Turrittin, Inst. Hautes Études Sci. Publ. Math. 39 (1970), 175-232.

[5] F. LiU and B. Osserman, Mochizuki's indigenous bundles and Ehrhart polynomials, J. Algebraic Combin. 23 (2006), 125-136.

[6] S. Mochizuki, A theory of ordinary p-adic curves, Publ. Res. Inst. Math. Sci. 32 (1996), 957-1152.

[7] S. MochizUKI, Foundations of $p$-adic Teichmüller theory, AMS/IP studies in advanced mathematics 11, American Mathematical Society, Providence, RI; International Press, Cambridge, MA, 1999.

[8] D. Mumford, Abelian varieties, Tata Institute of Fundamental Research studies in mathematics 5, Published for the Tata Institute of Fundamental Research, Bombay, Oxford University Press, London, 1970. 
NILPOTENT ADMISSIBLE INDIGENOUS BUNDLES IN CHARACTERISTIC THREE 731

[9] M. RaYnaud, Sections des fibrés vectoriels sur une courbe, Bull. Soc. Math. France 110 (1982), 103-125.

[10] M. Raynaud, Revêtements des courbes en caractéristique $p>0$ et ordinarité, Compositio Math. 123 (2000), 73-88.

[11] Y. WAKABAyashi, An explicit formula for the generic number of dormant indigenous bundles, Publ. Res. Inst. Math. Sci. 50 (2014), 383-409.

Yuichiro Hoshi

Research Institute for Mathematical Sciences

KYOTO UNIVERSITY

КYОТО 606-8502

JAPAN

E-mail: yuichiro@kurims.kyoto-u.ac.jp 